TRANSACTIONS OF THE

AMERICAN MATHEMATICAL SOCIETY

Volume 359, Number 1, January 2007, Pages 349-383

S 0002-9947(06)04180-8

Article electronically published on August 24, 2006

\title{
THE BLOCK STRUCTURE SPACES OF REAL PROJECTIVE SPACES AND ORTHOGONAL CALCULUS OF FUNCTORS
}

\author{
TIBOR MACKO
}

\begin{abstract}
Given a compact manifold $X$, the set of simple manifold structures on $X \times \Delta^{k}$ relative to the boundary can be viewed as the $k$-th homotopy group of a space $\widetilde{\mathcal{S}}^{s}(X)$. This space is called the block structure space of $X$.

We study the block structure spaces of real projective spaces. Generalizing Wall's join construction we show that there is a functor from the category of finite-dimensional real vector spaces with inner product to the category of pointed spaces which sends the vector space $V$ to the block structure space of the projective space of $V$. We study this functor from the point of view of orthogonal calculus of functors; we show that it is polynomial of degree $\leq 1$ in the sense of orthogonal calculus.

This result suggests an attractive description of the block structure space of the infinite-dimensional real projective space via the Taylor tower of orthogonal calculus. This space is defined as a colimit of the block structure spaces of projective spaces of finite-dimensional real vector spaces and is closely related to some automorphisms spaces of real projective spaces.
\end{abstract}

\section{INTRODUCTION}

The central objects of study in this paper are the block structure spaces of certain topological manifolds. These spaces arise as follows. For a closed topological manifold $X$, a simple manifold structure on $X$ is a simple homotopy equivalence $f$ from some closed manifold $M$ to $X$. Two simple manifold structures $f_{0}: M_{0} \rightarrow X$ and $f_{1}: M_{1} \rightarrow X$ are equivalent if there exists a homeomorphism $g: M_{0} \rightarrow M_{1}$ and a homotopy from $f_{1} \circ g$ to $f_{0}$. The set of equivalence classes is called the simple structure set of $X$ and is denoted by $\mathbb{S}^{s}(X)$. More generally, if $X$ is a compact manifold with boundary, there is a simple structure set $\mathbb{S}_{\partial}^{s}(X)$ whose elements are represented by simple homotopy equivalences of pairs $f:(M, \partial M) \rightarrow(X, \partial X)$ where the restricted map $\partial M \rightarrow \partial X$ is a homeomorphism.

For a given compact manifold $X$, Quinn constructed in $\mathrm{Qu}$ a space $\widetilde{\mathcal{S}}^{s}(X)$, the block structure space of $X$, such that

$$
\pi_{k}\left(\widetilde{\mathcal{S}}^{s}(X)\right) \cong \mathbb{S}_{\partial}^{s}\left(X \times \Delta^{k}\right) .
$$

This space is related to the automorphism spaces of $X$ via the homotopy fibration sequence

$$
\widetilde{\operatorname{TOP}}(X) \rightarrow \mathrm{G}^{s}(X) \rightarrow \widetilde{\mathcal{S}}^{s}(X)
$$

Received by the editors November 19, 2004.

2000 Mathematics Subject Classification. Primary 57N99, 55P99; Secondary 57R67.

Key words and phrases. The block structure space, manifold structure, join construction, orthogonal calculus, the first derivative functor, splitting problem, surgery.

(c)2006 American Mathematical Society Reverts to public domain 28 years from publication 
Here the space $\widetilde{\mathrm{TOP}}(X)$ is the block version of the space $\operatorname{TOP}(X)$ of self-homeomorphisms of $X$ and $\mathrm{G}^{s}(X)$ is the space of simple self-homotopy equivalences of $X$. See WW], BLR.

The simple structure sets $\mathbb{S}^{s}(X)$ have been studied in many special cases. In particular they were calculated for real projective spaces, lens spaces and complex projective spaces in the work of many authors, especially Wall Wa, chapter 14], Browder $[\mathrm{Br}$, Browder-Livesay $[\mathrm{BL}$ and López de Medrano $[\mathrm{LdM}]$. A useful tool used by these authors is the join construction (see [Wa, chapter 14]). It provides us with maps between simple structure sets in different dimensions. For example in the case of real projective spaces we get maps $\mathbb{S}^{s}\left(\mathbb{R} P^{n}\right) \rightarrow \mathbb{S}^{s}\left(\mathbb{R} P^{n+m}\right)$. We generalize the join construction to define maps between the block structure spaces.

In principle the homotopy type of the block structure space of any manifold is well understood as the homotopy fiber in the Sullivan-Wall-Quinn-Ranicki homotopy fibration sequence of surgery (see [WW, Theorem 2.3.1.]). Unfortunately, if $m>2$ this description of the block structure space does not easily extend to an illuminating description of the maps $\widetilde{\mathcal{S}}^{s}\left(\mathbb{R} P^{n}\right) \rightarrow \widetilde{\mathcal{S}}^{s}\left(\mathbb{R} P^{n+m}\right)$ induced by the join construction (Ranicki showed me how to handle the cases $m=1,2$ ). We propose to remedy this defect by using ideas from functor calculus.

For this purpose we formulate the join construction as a certain continuous functor. In the case of real projective spaces it is a functor, which we denote $F$, from the category $\mathcal{J}$ of real finite-dimensional vector spaces with inner product to the category Spaces $_{*}$ of pointed spaces which sends the vector space $V$ to the block structure space of $\mathbb{R} P(V)$, the real projective space of $V$. There are variants of this for the lens spaces and complex projective spaces. The general methods we use apply to all cases. However, in this paper we specialize to the case of real projective spaces. We make remarks on the other cases at the end of the Introduction.

The advantage of the formulation of the join construction as a continuous functor is that it enables us to use the orthogonal calculus of functors to study the block structure spaces of real projective spaces. The orthogonal calculus provides us with a general theory for studying continuous functors from $\mathcal{J}$ (as above) to $\mathrm{Spaces}_{*}$. It was developed by Weiss in We. He associates to a given continuous functor $E$ the tower of functors

$$
\cdots \rightarrow T_{k} E \rightarrow T_{k-1} E \rightarrow \cdots \rightarrow T_{0} E,
$$

called the Taylor tower. For each $k$ the functor $T_{k} E$ is a polynomial functor of degree $\leq k$ in a certain sense, and it is also the best approximation of $E$ by such a functor. Therefore it should be thought of as the $k$-th Taylor polynomial of $E$. For example, the functor $T_{0} E$ is defined by $T_{0} E(V)=\operatorname{hocolim}_{n} E\left(V \oplus \mathbb{R}^{n}\right)$. It is the best approximation of $E$ by an essentially constant functor (all induced maps $T_{0} E(V) \rightarrow T_{0} E(W)$ are homotopy equivalences). Thus the expansion is at infinity. The functor $E$ is related to its Taylor tower via natural transformations $E \rightarrow T_{k} E$ which fit together to form a commutative diagram. In favorable circumstances the maps $E(V) \rightarrow T_{k} E(V)$ are highly connected for $k$ large.

The idea of the calculus of functors is to obtain some information about the functor $E$ by investigating its Taylor tower. For this purpose we need to estimate the connectivity of the maps $E(V) \rightarrow T_{k} E(V)$. The best situation happens if the functor $E$ is polynomial of some degree $\leq k$. Then the maps $E(V) \rightarrow T_{k} E(V)$ are homotopy equivalences for all $V \in \mathcal{J}$. 
A continuous functor $E$ is called polynomial of degree $\leq k$ if the canonical map

$$
E(V) \rightarrow \operatorname{holim}_{0 \neq U \subseteq \mathbb{R}^{k+1}} E(V \oplus U)
$$

is a homotopy equivalence for all $V \in \mathcal{J}$. The homotopy limit is over the topological poset of all non-zero vector subspaces of $\mathbb{R}^{k+1}$ (in section 3 we give some details about homotopy limits in this setting). There is a way to verify that a given functor is polynomial of degree $\leq k$; in the case $k=1$ we discuss it in detail in the paper.

Once the convergence question is established (that means the connectivity of maps $E(V) \rightarrow T_{k} E(V)$ is known), the goal is to determine the Taylor tower itself. This can be done via an important theorem in We which says that the difference, hofiber $_{*}\left(T_{k} E(V) \rightarrow T_{k-1} E(V)\right)$, between the stages of the Taylor tower of the functor $E$ is determined by a certain spectrum $\Theta E^{(k)}$ with an action of the orthogonal group $O(k)$. This spectrum should therefore be thought of as the $k$-th derivative of $E$ at infinity.

The main theorem of this paper says that the functor $F$ defined above is essentially polynomial of degree $\leq 1$. It can be formulated as follows.

Theorem 5.1, Let $F: \mathcal{J} \rightarrow$ Spaces $_{*}$ be the functor defined by $V \mapsto \widetilde{\mathcal{S}}^{s}(\mathbb{R} P(V))$. Then the canonical maps

$$
F(V) \rightarrow \operatorname{holim}_{0 \neq U \subseteq \mathbb{R}^{2}} F(V \oplus U)
$$

are homotopy equivalences if $\operatorname{dim}(V) \geq 6$.

This implies that the maps $F(V) \rightarrow T_{1} F(V)$ are homotopy equivalences if $\operatorname{dim}(V) \geq 6$ (see Corollary [5.2) and therefore in high enough dimensions the Taylor tower of $F$ has only two interesting stages, namely $T_{1} F$ and $T_{0} F$. As mentioned above, the difference between them is measured by the first derivative of $F$ at infinity, which is a certain spectrum $\Theta F^{(1)}$ with an action of the orthogonal group $O(1)$. The homotopy groups of the spectrum $\Theta F^{(1)}$ are also determined.

Theorem 5.11, We have

$$
\pi_{k}\left(\Theta F^{(1)}\right) \cong L_{k}(1) \text { for } k \in \mathbb{Z},
$$

where the groups $L_{k}(1)$ are the 4-periodic L-groups from surgery theory associated to the trivial group.

There is another spectrum $\mathbf{L}_{\bullet}$ whose homotopy groups are $\pi_{k}\left(\mathbf{L}_{\bullet}\right) \cong L_{k}(1)$ for $k \in \mathbb{Z}$ (see [Ra2, chapter 13]). The relationship between $\boldsymbol{\Theta} F^{(1)}$ and $\mathbf{L}_{\bullet}$ is not yet clear to us, because we do not know any map between the two spectra.

The results of Theorems 5.1 and 5.11 can be used to provide a functorial description of spaces $F(V) \simeq T_{1} F(V)$ via the Taylor tower of $F$, if $\operatorname{dim}(V) \geq 6$. For example by comparing the Taylor tower of $F$ evaluated at 0 and at $V$ we obtain the homotopy fibration sequence

$$
\Omega^{\infty}\left[\left(S(V)_{+} \wedge \Theta F^{(1)}\right)_{h O(1)}\right] \rightarrow T_{1} F(0) \rightarrow T_{1} F(V),
$$

where $S(V)$ is the unit sphere in $V$ with the antipodal involution, the subscript + denotes added base point and the subscript $h O(1)$ denotes the homotopy orbit spectrum. We expect to determine the homotopy type of $T_{1} F(0)$ and the first map of the homotopy fibration sequence (0.3) jointly with Weiss in $\mathrm{MW}$. In any case, 
this yields a surprising similarity of the homotopy fibration sequence (0.3) with the Sullivan-Wall-Quinn-Ranicki homotopy fibration sequence of surgery theory for $\mathbb{R} P(V)$ (see $[\mathrm{WW}$, Theorem 2.3.1]). However, an important difference between the two homotopy fibration sequences is that $(0.3)$ is functorial in $V$, whereas the surgery theoretic description is NOT.

The Taylor tower of $F$ can also be used to give a satisfying description of the block structure spaces of the infinite-dimensional real projective space, that means the colimit

$$
F\left(\mathbb{R}^{\infty}\right)=\operatorname{colim}_{n \in \mathbb{N}} F\left(\mathbb{R}^{n}\right) \simeq T_{0} F(0) .
$$

Namely, the Taylor tower of $F$ evaluated at 0 becomes the homotopy fibration sequence

$$
\Omega^{\infty}\left[\left(\boldsymbol{\Theta} F^{(1)}\right)_{h O(1)}\right] \rightarrow T_{1} F(0) \rightarrow T_{0} F(0) \simeq F\left(\mathbb{R}^{\infty}\right) .
$$

If the promised results of [MW] are obtained, this yields the desired description of $F\left(\mathbb{R}^{\infty}\right)$.

The ultimate goal in the study of the block structure space of a manifold $X$ is to provide some information about the automorphism spaces of $X$, the space of self-homeomorphisms $\operatorname{TOP}(X)$ and the space of simple self-homotopy equivalences $\mathrm{G}^{s}(X)$. The relation between these spaces is via the homotopy fibration sequence (0.2), which involves the block version of the space of self homeomorphisms $\widetilde{\mathrm{TOP}}(X)$. If $A(X)$ is any of the automorphism spaces above, the join construction provides us with maps $A\left(\mathbb{R} P\left(\mathbb{R}^{n}\right)\right) \rightarrow A\left(\mathbb{R} P\left(\mathbb{R}^{m}\right)\right)$ for $n \leq m$, and the automorphism space $A\left(\mathbb{R} P^{\infty}\right)$ ) of the infinite-dimensional real projective space is defined as the colimit of $A\left(\mathbb{R} P\left(\mathbb{R}^{n}\right)\right)$ over $n \in \mathbb{N}$. The join maps commute with the maps in (0.2), and so we obtain the homotopy fibration sequence

$$
\left.\left.\left.\widetilde{\mathrm{TOP}}\left(\mathbb{R} P^{\infty}\right)\right) \rightarrow \mathrm{G}^{s}\left(\mathbb{R} P^{\infty}\right)\right) \rightarrow \widetilde{\mathcal{S}}^{s}\left(\mathbb{R} P^{\infty}\right)\right)=F\left(\mathbb{R}^{\infty}\right) .
$$

Closely related to these spaces are also certain equivariant automorphism spaces discussed in a more detail below. We remark that the space $\operatorname{colim}_{n \in \mathbb{N}} \mathrm{G}_{\mathbb{Z}_{2}}^{s}(S(n \cdot V))$ defined below is a trivial double cover of $\left.\mathrm{G}^{s}\left(\mathbb{R} P^{\infty}\right)\right)$, and in $[\mathrm{BS}$ ] it is shown that this space is homotopy equivalent to $Q\left(\mathbb{R} P_{+}^{\infty}\right)$, where $Q=\Omega^{\infty} \Sigma^{\infty}$ and the subscript + denotes the added base point. Therefore any information about the homotopy type of $F\left(\mathbb{R}^{\infty}\right)$ gives us also information about $\left.\widetilde{\mathrm{TOP}}\left(\mathbb{R} P^{\infty}\right)\right)$ which is closely related to $\operatorname{TOP}\left(\mathbb{R} P^{\infty}\right)$ ) (see $[\mathrm{WW}$ ] for more information about the relation between various automorphism spaces, also note that for real projective spaces the word simple can be dropped as $\left.\mathrm{Wh}\left(\mathbb{Z}_{2}\right)=\{0\}\right)$.

There is also a more direct way to relate the space $F\left(\mathbb{R}^{\infty}\right)$ to certain honest (not block) equivariant automorphism spaces as follows. Let $W$ be the regular representation of the group $\mathbb{Z}_{2}$. Denote by $\mathrm{G}_{\mathbb{Z}_{2}}(S(n \cdot W))$ the topological monoid of equivariant self-homotopy equivalences of the unit sphere $S(n \cdot W)$ of the induced representation $n \cdot W=\mathbb{R}^{n} \otimes W$ and by $\mathrm{TOP}_{\mathbb{Z}_{2}}(S(n \cdot W))$ the topological group of equivariant self-homeomorphisms of $S(n \cdot W)$. The join map induces inclusions $\mathrm{G}_{\mathbb{Z}_{2}}(S(n \cdot W)) \hookrightarrow \mathrm{G}_{\mathbb{Z}_{2}}(S(m \cdot W))$ and $\operatorname{TOP}_{\mathbb{Z}_{2}}(S(n \cdot W)) \hookrightarrow \mathrm{TOP}_{\mathbb{Z}_{2}}(S(m \cdot W))$ for $n \leq m$, and there are also inclusions $\operatorname{TOP}_{\mathbb{Z}_{2}}(S(n \cdot W)) \hookrightarrow \mathrm{G}_{\mathbb{Z}_{2}}(S(n \cdot W))$ which commute with the join map. Therefore we get the maps between quotients induced by the join construction. After passing to the colimit over $n \in \mathbb{N}$ there is the 
following splitting:

$$
\underset{n \in \mathbb{N}}{\operatorname{colim}} \mathrm{G}_{\mathbb{Z}_{2}}(S(n \cdot W)) / \mathrm{TOP}_{\mathbb{Z}_{2}}(S(n \cdot W)) \simeq F\left(\mathbb{R}^{\infty}\right)_{1} \times \mathrm{G} / \mathrm{TOP},
$$

which relates the left-hand side to the space $F\left(\mathbb{R}^{\infty}\right)$. Here $F\left(\mathbb{R}^{\infty}\right)_{1}$ is the base point component. For the classical space G/TOP see e.g. [WW, section 1].

Here is a sketch of how the splitting (0.6) is obtained. Let $W=V \oplus U$, where $V$ is the free part of the representation and $U$ is the trivial part. There is the splitting

$$
\underset{n \in \mathbb{N}}{\operatorname{colim}} \mathrm{G}_{\mathbb{Z}_{2}}(S(n \cdot W)) \simeq \underset{n \in \mathbb{N}}{\operatorname{colim}} \mathrm{G}_{\mathbb{Z}_{2}}(S(n \cdot V)) \times \underset{n \in \mathbb{N}}{\operatorname{colim}} \mathrm{G}(S(n \cdot U)) .
$$

See [Cr, Proposition 3.5] for the statement on the level of homotopy groups. Similarly there is the splitting

$$
\underset{n \in \mathbb{N}}{\operatorname{colim}} \operatorname{TOP}_{\mathbb{Z}_{2}}(S(n \cdot W)) \simeq \underset{n \in \mathbb{N}}{\operatorname{colim}} \widetilde{\mathrm{TOP}}_{\mathbb{Z}_{2}}(S(n \cdot V)) \times \underset{n \in \mathbb{N}}{\operatorname{colim}} \operatorname{TOP}(S(n \cdot U)) .
$$

This is obtained by passing to the colimit over $n$ with the splittings

$$
\mathrm{TOP}_{\mathbb{Z}_{2}}(S(n \cdot W)) \simeq \mathrm{TOP}_{\mathbb{Z}_{2}}(S(n \cdot W), \text { rel } S(n \cdot U)) \times \operatorname{TOP}(S(n \cdot U)),
$$

where rel $S(n \cdot U)$ means that the equivariant self-homeomorphisms of $S(n \cdot W)$ are fixed to be the identity on $S(n \cdot U) \hookrightarrow S(n \cdot W)$. The splitting (0.9) is analogous to the splitting in $[\mathrm{Cr}$, Lemma 3.4]. The second factor in (0.8) is the colimit of the second factors of ([0.9). The first factor of (0.8) is obtained by relating the first factor of (0.9) to the equivariant bounded self-homeomorphisms of $S(n \cdot V) \times(n \cdot U)$ using a trick of [AH, section 3] and then relating the bounded self-homeomorphisms to block self-homeomorphisms as in WW, Proposition 1.4.2]. Finally, these equivariant automorphism spaces of $S(n \cdot V)$ map into automorphism spaces of $\mathbb{R} P(n \cdot V)$ by passing to quotients, and this map is a trivial double cover. The splitting (0.6) then follows from (0.5) because the join maps commute with the splittings (0.7), (0.8) and we have

$$
\begin{aligned}
& \mathrm{G}_{\mathbb{Z}_{2}}(S(n \cdot V)) / \widetilde{\mathrm{TOP}}_{\mathbb{Z}_{2}}(S(n \cdot V)) \cong \\
& \mathrm{G}(\mathbb{R} P(n \cdot V)) / \widetilde{\mathrm{TOP}}(\mathbb{R} P(n \cdot V)) \cong \widetilde{\mathcal{S}}_{1}^{s}(\mathbb{R} P(n \cdot V)) .
\end{aligned}
$$

Now we would like to briefly discuss the study of the block structure spaces of lens spaces and complex projective spaces. Using the join construction we obtain in these cases a functor from the category of complex vector spaces with inner product to Spaces $_{*}$ which sends the vector space $V$ to $\widetilde{\mathcal{S}}^{s}(L(V))$ or $\widetilde{\mathcal{S}}^{s}(\mathbb{C} P(V))$. Here we can employ the unitary calculus which is the complex analogue of orthogonal calculus, and we can try to run the study parallel to the case of real projective spaces. The case of lens spaces was studied by Madsen and Rothenberg in MR, where they show that the join maps induce isomorphisms on homotopy groups (they also determine the homotopy groups, and later Madsen in Mad determines the homotopy type localized at 2 and away from 2). Hence, from our point of view, the corresponding functor is polynomial of degree 0 . The case of complex projective spaces is not covered in $[\mathrm{MR}$, so the parallel study would be interesting. We note that the main ingredient in the proofs of Theorems 5.1 and 5.11 is the codimension 1 surgery theory, whereas in the case of complex projective spaces we have to use 
codimension 2 surgery theory, which is considerably more complicated (see Ra1, chapter 7.8]). At the moment we are unable to carry over the whole proofs of Theorems 5.1, 5.11. We hope to do this in the future.

The paper is organized as follows. In section 1 we introduce the block structure spaces. In section 2 we construct the functor $F$. In section 3 we give an overview of the basic constructions from orthogonal calculus that we use in the paper. In particular for a continuous functor $E: \mathcal{J} \rightarrow$ Spaces $_{*}$ we recall a necessary and sufficient condition for $E$ to be polynomial of degree $\leq 1$ (Theorem 3.6). This involves studying a certain functor $E^{(1)}: \mathcal{J} \rightarrow$ Spaces $_{*}$ with some additional structure, which is called the first derivative functor of $E$. Section 4 contains background material about codimension 1 surgery theory, which is used to give a description of the first derivative functor $F^{(1)}$. Up to now we claim no originality. The description of $F^{(1)}$ will then be the main tool in the proofs of Theorems 5.1 and 5.11 which are given in section 5. The proofs are our contribution, and they are the core of the paper. We note that section 3 is a short survey of orthogonal calculus, however, for complete information we refer the reader to the original source We. Throughout the paper we also assume basic knowledge of surgery theory (that means the book Wa up to chapter 10), but we provide some background about the less standard parts, such as codimension 1 surgery theory.

Notation 0.1 . For $V \in \mathcal{J}$, by $\mathbb{R} P(V)$ we denote the real projective space of $V$, by $S(V, r)$ we denote the sphere with radius $r \geq 0$ in $V$, and $S(V)=S(V, 1)$.

\section{The BLOCK STRUCTURE SPACE OF A MANIFOLD}

In this section we introduce the block structure space of a closed manifold $X$ in general and we also introduce a method for constructing other models for the block structure spaces. The method will be used in section 2 to obtain a functorial model of the block structure spaces of the real projective spaces.

In the Introduction we have defined for a given closed manifold $X$ its simple structure set $\mathbb{S}^{s}(X)$ and more generally for a compact manifold with boundary $(X, \partial X)$ the simple structure set $\mathbb{S}_{\partial}^{s}(X)$. These are the basic objects of study of surgery theory which have been calculated in many special cases (see for example Wa], [LdM]).

For a compact manifold $X$ the structure sets $\mathbb{S}_{\partial}^{s}\left(X \times \Delta^{k}\right)$ become groups when $k \geq 1$. The block structure space of $X$ is designed to be a space such that

$$
\pi_{k}\left(\widetilde{\mathcal{S}}^{s}(X)\right) \cong \mathbb{S}_{\partial}^{s}\left(X \times \Delta^{k}\right) .
$$

It is constructed as a $\Delta$-set (see [RS]). If $X$ is a closed manifold, then a $k$-simplex of $\widetilde{\mathcal{S}}^{s}(X)$ is a simple homotopy equivalence $f: M \rightarrow X \times \Delta^{k}$ between $(n+k)$ dimensional manifold $(k+2)$-ads, where $n=\operatorname{dim}(X)$ (the notion of a manifold $(k+2)$-ad is discussed below). The face operators are given by restrictions, and the base point in dimension $k$ is id: $X \times \Delta^{k} \rightarrow X \times \Delta^{k}$.

The construction of the space $\widetilde{\mathcal{S}}^{s}(X)$ was given by Quinn in $\mathrm{Qu}$. As indicated in the Introduction the main motivation for studying this space comes from the fact that it is closely related to the automorphism spaces of $X$. We refer the reader to the survey article [WW] for more information about this application. In [WW, Theorem 2.3.1] it is also stated that $\widetilde{\mathcal{S}}^{s}(X)$ fits into the homotopy fibration sequence of surgery theory of Sullivan-Wall-Quinn-Ranicki, which in principle determines its homotopy type. 
As already mentioned, when $X$ is the real projective space $\mathbb{R} P(V)$ for some $V \in \mathcal{J}$, we obtain an additional structure of maps between the block structure spaces of real projective spaces in different dimensions. These maps, called the join maps, were described on the homotopy groups by Wall Wa for $\pi_{0}$ and by Madsen and Rothenberg [MR] for $\pi_{k}$, when $k \geq 1$. We generalize these maps to the level of spaces in a functorial way. For this we need another model of the block structure space of $\mathbb{R} P(V)$, that means a space homotopy equivalent to $\widetilde{\mathcal{S}}^{s}(\mathbb{R} P(V))$. The method for constructing such a space, which we describe in this section, is based on a careful analysis of Quinn's model. The desired model is then constructed in two steps. In this section we construct an intermediate model $\widetilde{\mathcal{S}}_{\mathbb{Z}_{2}}^{s}(S(V))$, which also admits the join maps, as explained in MR. However, these do not define a functor because they do not behave well w.r.t. the composition. Then, in the next section, we construct the functorial model $\widetilde{\mathcal{S}}_{\mathbb{Z}_{2}}^{s}(V)$.

Remark 1.1. Each of the models $\widetilde{\mathcal{S}}^{s}(\mathbb{R} P(V)), \widetilde{\mathcal{S}}_{\mathbb{Z}_{2}}^{s}(S(V)), \widetilde{\mathcal{S}}_{\mathbb{Z}_{2}}^{s}(V)$ has convenient properties in different situations. As they are all homotopy equivalent (at least in high dimensions) and as many of the results we need are only 'up to homotopy' we switch between these models throughout the paper according to our needs.

First we elaborate on the definition of a manifold $(k+2)$-ad. It is a generalization of a cobordism between manifolds, namely a cobordism is a special manifold 3-ad. According to $\mathrm{Mi}$ ( or $[\mathrm{Lu}]$ ) a cobordism between two $n$-dimensional manifolds $M_{0}$, $M_{1}$ is a 5 -tuple $\left(M, M_{0}, M_{1}, h_{0}, h_{1}\right)$, where $M$ is an $(n+1)$-dimensional manifold with two disjoint pieces of boundary $\partial_{0} M, \partial_{1} M$ and $h_{i}: M_{i} \rightarrow \partial_{i} M$ for $i=0,1$ are homeomorphisms of $n$-dimensional manifolds. A manifold $(k+2)$-ad is a generalization where the manifold $M$ has $(k+1)$ pieces of boundary which fit together in a nice way. We formalize this notion below.

More precisely we give an abstract construction of a $\Delta$-set which consists of objects formally resembling manifold $(k+2)$-ads as an associated $\Delta$-set to a certain $\Delta$-groupoid. This is done in Construction 1.3 and then applied to the special case of the block structure spaces in Construction 1.6.

We recall basic definitions for $\Delta$-sets of [RS] (see also [Ra2, chapter 11]). A $\Delta$-set $K$ is a functor $K: \Delta^{o p} \rightarrow$ Sets, where $\Delta$ is the category with objects finite totally ordered sets $\mathbf{k}=\{0, \ldots, k\}$ and morphisms injective monotone functions $\alpha: \mathbf{m} \rightarrow \mathbf{k}$ for $m \leq k$. Thus a $\Delta$-set $K$ can be described as a collection of sets $\left\{K_{k}\right\}_{k \in \mathbb{N}}$ together with face maps $\partial_{\alpha}: K_{k} \rightarrow K_{m}$. The standard $k$-simplex is a $\Delta$ set in the following way: the set of $m$-simplices $\Delta_{m}^{k}$ is the set of monotone injective functions $\sigma: \mathbf{m} \rightarrow \mathbf{k}$, and for $\beta: \mathbf{l} \rightarrow \mathbf{m}$ the face map $\partial_{\beta}: \Delta_{m}^{k} \rightarrow \Delta_{l}^{k}$ is defined by $\partial_{\beta}(\sigma)=\sigma \circ \beta$. Also $\alpha: \mathbf{m} \rightarrow \mathbf{k}$ defines a map of $\Delta$-sets $\Delta^{m} \rightarrow \Delta^{k}$. More generally, a $\Delta$-groupoid $K$ is a functor $K: \Delta^{o p} \rightarrow$ Groupoids, that means for each $k$ the set of $k$-simplices $K_{k}$ is a groupoid (a category where all morphisms are isomorphisms) and the face maps $\partial_{\alpha}$ are functors. Note that the standard simplex $\Delta^{k}$ is also a $\Delta$-groupoid in a trivial way. A $\Delta$-set $K$ is pointed if for every $k \in \mathbb{N}$ there is a base simplex $* \in K_{k}$ such that $\partial_{\alpha} *=*$ for all $\alpha$.

Now we recall the following well-known construction for the special case of a $\Delta$-groupoid.

Construction $1.2(\overline{\mathrm{Dw}})$. Let $K$ be a $\Delta$-groupoid. The Grothendieck construction $\operatorname{Gr}(K)$ of $K$ is the category with objects pairs $(\mathbf{k}, x)$, where $\mathbf{k} \in \Delta, x \in K_{k}$, and morphisms $(\mathbf{k}, x) \rightarrow(\mathbf{m}, y)$ are pairs $(\alpha, h)$, where $\alpha: \mathbf{m} \rightarrow \mathbf{k}$ is a morphism in $\Delta$ 
and $h: \partial_{\alpha} x \rightarrow y$ is a morphism in $K_{m}$. We also define the degree of the object $(\mathbf{k}, x)$ to be $k \in \mathbb{N}$. Note that the construction is functorial. In particular for $\alpha: \mathbf{m} \rightarrow \mathbf{k}$ we have a functor $\operatorname{Gr}(\alpha): \operatorname{Gr}\left(\Delta^{m}\right) \rightarrow \operatorname{Gr}\left(\Delta^{k}\right)$.

Construction 1.3. Let $K$ be a $\Delta$-groupoid. We define the associated $\Delta$-set of $K$ to be the $\Delta$-set $K^{\sharp}$ defined as follows. The set of $k$-simplices of the $\Delta$-set $K^{\sharp}$ is the set of degree-preserving functors $\sigma: G r\left(\Delta^{k}\right) \rightarrow G r(K)$ which satisfy the extra condition that for $\beta: \mathbf{l} \rightarrow \mathbf{m}$ the morphism $\sigma(\beta, i d)$ equals $\left(\beta, h_{\beta}\right)$ for some $h_{\beta} \in K_{l}$. For $\alpha: \mathbf{m} \rightarrow \mathbf{k}$ the face map $\partial_{\alpha}^{\sharp}: K_{k}^{\sharp} \rightarrow K_{m}^{\sharp}$ is defined by $\partial_{\alpha}^{\sharp} \sigma=\sigma \circ G r(\alpha)$.

A $k$-simplex $\sigma \in K^{\sharp}$ can be described by a list $\sigma=\left(x_{\alpha}, h_{\alpha}\right)$, where $x_{\alpha} \in K_{m}$ and $h_{\alpha}: \partial_{\alpha} x \rightarrow x_{\alpha}$ is an isomorphism in $K_{m}$ for $\alpha: \mathbf{m} \rightarrow \mathbf{k}$ a morphism in $\Delta$, satisfying various compatibility conditions. Here $x=x_{\text {id }}$. Note that if $K$ is a trivial $\Delta$-groupoid (all morphisms are the identity), then a $k$-simplex of $K^{\sharp}$ is just a collection $\sigma=\left(x_{\alpha}\right.$,id), where $x_{\alpha}=\partial_{\alpha} x$. Thus we recover the underlying $\Delta$-set of $K$. If $K$ is a non-trivial $\Delta$-groupoid a $k$-simplex of $K^{\sharp}$ is a collection $\sigma=\left(x_{\alpha}, h_{\alpha}\right)$, where objects $x_{\alpha}$ are just isomorphic to $\partial_{\alpha} x$.

Remark 1.4. The assignment $K \mapsto K^{\sharp}$ is a functor from $\Delta$-groupoids to $\Delta$-sets. Suppose that $K$ is a topological $\Delta$-groupoid, that means the category $K_{k}$ is a topological category for each $k$ and the face operators are continuous functors. Then $K_{k}^{\sharp}$ can also be endowed with a topology (the subspace topology of the product topology) and $K^{\sharp}$ becomes a $\Delta$-space, also in a functorial way. The geometric realization then converts the $\Delta$-set (or the $\Delta$-space) $K^{\sharp}$ into a genuine space again in a functorial way. In the notation we do not distinguish between the underlying $\Delta$ set (or $\Delta$-space) and its geometric realization. We note that all these constructions have pointed versions.

Definition 1.5. A $k$-block $M$ is a manifold with boundary such that the boundary $\partial M$ is decomposed into codimension 0 submanifolds $\partial_{i} M$, for $i \in \mathbf{k}$, whose intersections $\partial_{i} M \cap \partial_{j} M$ are codimension 0 submanifolds of the boundaries of $\partial_{i} M$ and $\partial_{j} M$, and so on. For $\alpha: \mathbf{m} \rightarrow \mathbf{k}$ a morphism in $\Delta$ we denote $\partial_{\alpha} M=\bigcap_{i \in \operatorname{im}(\alpha)} \partial_{i} M$. A block map $f: M \rightarrow N$ between two $k$-blocks is a map, such that $f\left(M_{\alpha}\right) \subset N_{\alpha}$ for $\alpha \in \mathbf{k}$. A $k$-block is special if $\bigcap_{i \in \mathbf{k}} \partial_{i} M=\emptyset$.

An example of a special $k$-block is $X \times \Delta^{k}$ for $X$ a closed manifold. Now we are ready for the definition of the block structure space $\widetilde{\mathcal{S}}^{s}(X)$.

Construction 1.6. Let $X$ be a closed $n$-dimensional manifold. The block structure space $\widetilde{\mathcal{S}}^{s}(X)$ of $X$ is the associated pointed $\Delta$-set of the pointed $\Delta$-groupoid whose $k$-simplices are pairs $(M, f)$, where $M$ is a special $k$-block, and a block map $f: M \rightarrow X \times \Delta^{k}$ is a simple homotopy equivalence between $(n+k)$-dimensional $k$-blocks. Face operators $\partial_{\alpha}$ are obtained by taking the face $\partial_{\alpha} M$ and restricting $f$ to $\left.f\right|_{\partial_{\alpha} M}: \partial_{\alpha} M \rightarrow X \times \partial_{\alpha} \Delta^{k}$. An isomorphism between $(M, f)$ and $\left(M^{\prime}, f^{\prime}\right)$ is a block homeomorphism $h: M \rightarrow M^{\prime}$ such that $f=f^{\prime} \circ h$. The base point in dimension $k$ is id: $X \times \Delta^{k} \rightarrow X \times \Delta^{k}$.

A $k$-simplex of $\widetilde{\mathcal{S}}^{s}(X)$ can be described by a list $\left(\left(M_{\alpha}, f_{\alpha}\right), h_{\alpha}\right)$, where for $\alpha: \mathbf{m} \rightarrow \mathbf{k}$ the $M_{\alpha}$ is a special $m$-block, the map $f_{\alpha}: M_{\alpha} \rightarrow X \times \partial_{\alpha} \Delta^{k}$ is a simple homotopy equivalence of $m$-blocks, $h_{\alpha}: M_{\alpha} \rightarrow \partial_{\alpha} M$ is a homeomorphism of $m$-blocks and the maps $f_{\alpha}, h_{\alpha}$ satisfy various compatibility conditions. The list 
$\left(M_{\alpha}, h_{\alpha}\right)$ is the correct formalization of a manifold $(k+2)$-ad. In the case $k=1$, the manifold 3 -ad is a cobordism in the sense of [Mi], $\mathrm{Lu}$ as described earlier.

The block structure space $\widetilde{\mathcal{S}}^{s}(X)$ as defined above is a Kan $\Delta$-set for any manifold $X$. For Kan $\Delta$-sets there is a convenient combinatorial formula for homotopy groups (see [Ra2, chapter 11]), and using it we immediately obtain the isomorphisms (1.1).

The following lemma provides us with a method for constructing other models of the block structure space.

Lemma 1.7. Let $L \rightarrow K$ be a map of pointed $\Delta$-groupoids such that $L^{\sharp}$ and $K^{\sharp}$ are Kan $\Delta$-sets. If $L_{k} \rightarrow K_{k}$ induces a bijection on equivalence classes of objects for every $k$, then the induced map $L^{\sharp} \rightarrow K^{\sharp}$ is a homotopy equivalence.

Proof. Using the combinatorial expression of $\pi_{k}$ it is easy to see that the map $L^{\sharp} \rightarrow K^{\sharp}$ induces isomorphisms on homotopy groups.

Now we specialize to the real projective spaces. Note first that any homotopy equivalence $f: M \rightarrow \mathbb{R} P(V) \times \Delta^{k}$ is simple because $\mathrm{Wh}\left(\mathbb{Z}_{2}\right)=\{0\}$ (see [Lu]). Therefore we drop the word simple when dealing with the real projective spaces. Here is the promised intermediate model $\widetilde{\mathcal{S}}_{\mathbb{Z}_{2}}^{s}(S(V))$ for the block structure space of $\mathbb{R} P(V)$.

Construction 1.8. Let $\widetilde{\mathcal{S}}_{\mathbb{Z}_{2}}^{s}(S(V))$ be the associated pointed $\Delta$-set of the pointed $\Delta$-groupoid whose $k$-simplex is a pair $(T, f)$, where:

- $T$ is a free involution on $S(V) \times \Delta^{k}$, which, as a map, is a block map,

- $f: S(V) \times \Delta^{k} \rightarrow S(V) \times \Delta^{k}$ is a block map which is an equivariant homotopy equivalence w.r.t. $T$ on the source and $T_{a}$ on the target, where $T_{a}$ is the product of the antipodal involution on $V$ (restricted to $S(V)$ ) with the identity on $\Delta^{k}$.

Face maps are defined by restriction, and an isomorphism between $(T, f)$ and $\left(T^{\prime}, f^{\prime}\right)$ is an equivariant block homeomorphisms $h: S(V) \times \Delta^{k} \rightarrow S(V) \times \Delta^{k}$ such that $f=f^{\prime} \circ h$. The base point is the pair $\left(T_{a}\right.$, id). A pair $(T, f)$ which represents a $k$-simplex in $\widetilde{\mathcal{S}}_{\mathbb{Z}_{2}}^{s}(S(V))$ will be shortly referred to as a structure on $S(V) \times \Delta^{k}$.

There is a map $\widetilde{\mathcal{S}}_{\mathbb{Z}_{2}}^{s}(S(V)) \rightarrow \widetilde{\mathcal{S}}^{s}(\mathbb{R} P(V))$ defined on the underlying $\Delta$-groupoids by taking quotients.

Proposition 1.9. The map $\widetilde{\mathcal{S}}_{\mathbb{Z}_{2}}^{s}(S(V)) \rightarrow \widetilde{\mathcal{S}}^{s}(\mathbb{R} P(V))$ is a homotopy equivalence if $\operatorname{dim}(V) \geq 5$.

Proof of Proposition 1.9. Using Lemma 1.7 it is enough to show that for each $k \in \mathbb{N}$ the map from the $k$-simplices of the underlying groupoid of $\widetilde{\mathcal{S}}_{\mathbb{Z}_{2}}^{s}(S(V))$ to the $k$ simplices of the underlying groupoid of $\widetilde{\mathcal{S}}^{s}(\mathbb{R} P(V))$ induces an isomorphism on the equivalence classes of objects. We proceed by induction on $k$. From the generalized Poincaré conjecture in $\operatorname{dim}(V) \geq 5$ we have that any manifold $M$ homotopy equivalent to $\mathbb{R} P(V)$ has the universal cover homeomorphic to $S(V)$ which proves the statement for $k=0$. For the induction step we apply the $h$-cobordism theorem which implies that the universal cover of any manifold $(k+2)$-ad $M$ mapping via a homotopy equivalence to $\mathbb{R} P(V) \times \Delta^{k}$ is homeomorphic to $S(V) \times \Delta^{k}$. 


\section{THE JOIN CONSTRUCTION}

In this section we construct a continuous functor $F: \mathcal{J} \rightarrow$ Spaces $_{*}$, such that for an object $V \in \mathcal{J}$ with $\operatorname{dim}(V) \geq 5$, the space $F(V)$ is homotopy equivalent to the space $\widetilde{\mathcal{S}}^{s}(\mathbb{R} P(V))$ and for a morphism $\xi \in \mathcal{J}$ the map $F(\xi)$ is a generalization of the join construction of Wall.

Construction 2.1. Given an object $V \in \mathcal{J}$, let $\widetilde{\mathcal{S}}_{\mathbb{Z}_{2}}^{s}(V)$ be the associated pointed $\Delta$-set of the pointed $\Delta$-groupoid whose $k$-simplex is a pair $(T, f)$ where:

- $T$ is an involution on $V \times \Delta^{k}$, which, as a map, is a block map, it leaves $S(V, r) \times \Delta^{k}$ invariant for each $r \geq 0$, its restriction to $S(V, r) \times \Delta^{k}$ is free for $r>0$, and its restriction to $0 \times \Delta^{k}$ is trivial,

- $f: V \times \Delta^{k} \rightarrow V \times \Delta^{k}$ is a block map, which is equivariant w.r.t. $T$ on the source and $T_{a}$ on the target, it leaves $S(V, r) \times \Delta^{k}$ invariant for each $r \geq 0$, the restriction of $f$ to $S(V, r) \times \Delta^{k}$ is an equivariant homotopy equivalence for $r>0$, and the restriction of $f$ to $0 \times \Delta^{k}$ is a homeomorphism.

Face maps are defined by restriction, an isomorphism from $(T, f)$ to $\left(T^{\prime}, f^{\prime}\right)$ is an equivariant block homeomorphism $h: V \times \Delta^{k} \rightarrow V \times \Delta^{k}$ such that $f=f^{\prime} \circ h$, and the base point is the pair $\left(T_{a}, \mathrm{id}\right)$. A pair $(T, f)$ which represents a $k$-simplex in $\widetilde{\mathcal{S}}_{\mathbb{Z}_{2}}^{s}(V)$ will be shortly referred to as a structure on $V \times \Delta^{k}$.

Construction 2.2. Given a morphism $\xi: V \rightarrow W$ in $\mathcal{J}$, let $\widetilde{\mathcal{S}}_{\mathbb{Z}_{2}}^{s}(\xi): \widetilde{\mathcal{S}}_{\mathbb{Z}_{2}}^{s}(V) \rightarrow$ $\widetilde{\mathcal{S}}_{\mathbb{Z}_{2}}^{s}(W)$ be a map defined as follows. The linear inclusion $\xi$ induces a direct sum decomposition $W=\xi(V) \oplus \xi(V)^{\perp}$ and the product decomposition $W \times \Delta^{k}=$ $\xi(V) \times \Delta^{k} \times \xi(V)^{\perp}$. First we obtain the structure $\left(\xi \circ T \circ \xi^{-1}, \xi \circ f \circ \xi^{-1}\right)$ on $\xi(V) \times \Delta^{k}$. The desired structure on $W \times \Delta^{k}$ is defined by

$$
\left(\xi \circ T \circ \xi^{-1}, \xi \circ f \circ \xi^{-1}\right) \times\left(T_{a}, \mathrm{id}\right)_{\xi(V)^{\perp}} .
$$

Theorem 2.3. Given an object $V \in \mathcal{J}$, let $F(V)$ be the geometric realization of $\widetilde{\mathcal{S}}_{\mathbb{Z}_{2}}^{s}(V)$, and given a morphism $\xi \in \mathcal{J}$, let $F(\xi)$ be the geometric realization of $\widetilde{\mathcal{S}}_{\mathbb{Z}_{2}}^{s}(\xi)$. Then $F$ defines a functor from $\mathcal{J}$ to Spaces $_{*}$.

The statement is easy to verify and we leave it for the reader. In order to show that the space $\widetilde{\mathcal{S}}_{\mathbb{Z}_{2}}^{s}(V)$ defines another model for the block structure space of $\mathbb{R} P(V)$ we use the method of the previous section to prove the following proposition.

Proposition 2.4. For each $V \in \mathcal{J}$ there exist maps

$$
\text { res: } \widetilde{\mathcal{S}}_{\mathbb{Z}_{2}}^{s}(V) \rightarrow \widetilde{\mathcal{S}}_{\mathbb{Z}_{2}}^{s}(S(V)) \quad \text { and } \quad \text { cone: } \widetilde{\mathcal{S}}_{\mathbb{Z}_{2}}^{s}(S(V)) \rightarrow \widetilde{\mathcal{S}}_{\mathbb{Z}_{2}}^{s}(V)
$$

which are homotopy inverses of each other.

Remark 2.5. As already indicated, throughout the paper we also work with the models $\widetilde{\mathcal{S}}^{s}(\mathbb{R} P(V))$ and $\widetilde{\mathcal{S}}_{\mathbb{Z}_{2}}^{s}(S(V))$, which is legal for the statements which are 'up to homotopy'. Similarly, we work with the join maps on these models. For example in section 5 the model for the join map is (res $\circ F(\xi) \circ$ cone).

Construction 2.6. The maps res and cone are induced by the maps between the corresponding pointed $\Delta$-groupoids. Since we think of $S(V) \times \Delta^{k}=S(V, 1) \times \Delta^{k}$ 
as a subspace of $V \times \Delta^{k}$, we can define

$$
\text { res: } \widetilde{\mathcal{S}}_{\mathbb{Z}_{2}}^{s}(V) \rightarrow \widetilde{\mathcal{S}}_{\mathbb{Z}_{2}}^{s}(S(V))
$$

by $(T, f) \mapsto\left(\left.T\right|_{\left(S(V) \times \Delta^{k}\right)},\left.f\right|_{\left(S(V) \times \Delta^{k}\right)}\right)$.

The map

$$
\text { cone: } \widetilde{\mathcal{S}}_{\mathbb{Z}_{2}}^{s}(S(V)) \rightarrow \widetilde{\mathcal{S}}_{\mathbb{Z}_{2}}^{s}(V)
$$

is defined by a certain 'iterated cone construction' which we now describe. Let $(T, f)$ be a structure on $S(V) \times \Delta^{k}$ and let $\left(T^{\prime}, f^{\prime}\right)=\operatorname{cone}(T, f)$, which is a structure on $V \times \Delta^{k}$. We describe a general procedure for constructing a block self-map of $V \times \Delta^{k}$, which preserves $S(V, r) \times \Delta^{k}$ for each $r \geq 0$, from a block self-map of $S(V) \times \Delta^{k}$. The maps $T^{\prime}, f^{\prime}$ are constructed from $T, f$ using this procedure.

The case $k=0$. Let $X$ be a topological space. The open cone on $X$ is the space $C^{+} X$ whose points are linear expressions $r x$, where $r \in[0, \infty)$. This includes the identifications $0=0 x=0 y$. The point 0 is called the cone point. We have a decomposition $C^{+} X=C X \cup C^{1+} X$, with $C X=\{r x \mid r \in[0,1]\}$, and $C^{1+} X=$ $\{r x \mid r \in[1, \infty)\}$. For $r \geq 0$ we distinguish the subspaces $r \cdot X=\{r x \mid x \in X\}$ and we identify $X=1 \cdot X$. Sometimes we want to give the cone point some other name than 0 , e.g. $c$. In that case the points of the subspace $C X$ are linear combinations $r x+(1-r) c$ for $x \in X$ and $r \in[0,1]$. Note that, if $X$ is $S(V)$, then $C^{+} X$ can be identified with $V$, so that $r \cdot X$ is identified with $S(V, r)$ for $r \geq 0$.

Let $h: X \rightarrow Y$ be a map. Then the rule $r x \mapsto r h(x)$ defines an extension $C^{+}(h)$ of $h$ such that $C^{+}(h)(r \cdot X) \subseteq r \cdot Y$.

The cases $k>0$. Let $h: X \times \Delta^{k} \rightarrow Y \times \Delta^{k}$ be a block map. We define its extension, a block map $C^{+}(h): C^{+} X \times \Delta^{k} \rightarrow Y \times \Delta^{k}$, such that $C^{+}(h)\left(r \cdot X \times \Delta^{k}\right) \subseteq r \cdot Y \times \Delta^{k}$.

We have the decomposition $C^{+} X \times \Delta^{k}=C X \times \Delta^{k} \cup C^{1+} X \times \Delta^{k}$. On $C^{1+} X \times \Delta^{k}$ the extension is defined as in the case $k=0$, i.e. if $h(x, s)=\left(h(x, s)_{1}, h(x, s)_{2}\right)$, then $C^{+}(h):(r x, s) \mapsto\left(r h(x, s)_{1}, h(x, s)_{2}\right)$. On $C X \times \Delta^{k}$ we use the following 'iterated cone construction'.

Starting with $X \times \Delta^{k}$ we define the iterated cone $C_{k}\left(X \times \Delta^{k}\right)$ by

$$
C_{k}\left(X \times \Delta^{k}\right)=C\left(X \times \Delta^{k} \cup \bigcup_{\tau} C_{k-1}(X \times \tau)\right),
$$

where $\tau<\Delta^{k},|\tau|=k-1$.

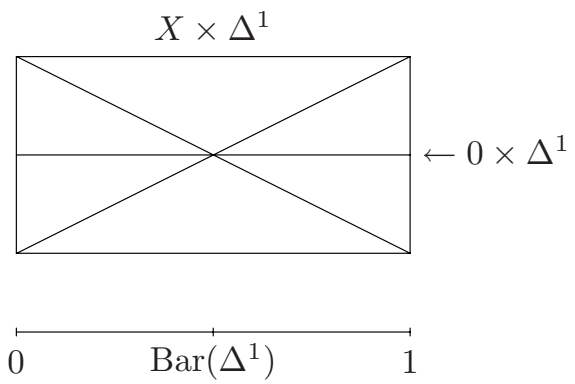

Figure 1. Picture of $C_{1}\left(X \times \Delta^{1}\right)$ for $X=S^{0}$.

Figure 1 depicts the special case when $X=S^{0}$ and $k=1$. (Here mutually distinct cone points should be used for different cones in sight.) 
The spaces $C X \times \Delta^{k}$ and $C_{k}\left(X \times \Delta^{k}\right)$ can be identified by choosing suitable cone points in the above description. Namely, if $\tau \leq \Delta^{k}$, then choose the cone point of $C_{|\tau|}(X \times \tau)$ to be the barycenter $\operatorname{Bar}(\tau)$. Explicitly, we obtain a homeomorphism $C_{|\tau|}(X \times \tau) \rightarrow C X \times \tau$ as follows. Suppose that for all $\sigma<\tau$ a homeomorphism $C_{|\sigma|}(X \times \sigma) \rightarrow C X \times \sigma$ was constructed. Then define a homeomorphism from $C(X \times \tau \cup C X \times \partial \tau)$ to $C X \times \tau$ by

$$
\left\{\begin{array}{l}
r(x, t)+(1-r) \operatorname{Bar}(\tau) \mapsto(r x, r t+(1-r) \operatorname{Bar}(\tau)), \text { if }(x, t) \in X \times \tau, \\
r(s x, t)+(1-r) \operatorname{Bar}(\tau) \mapsto(r s x, r t+(1-r) \operatorname{Bar}(\tau)), \text { if }(s x, t) \in C X \times \partial \tau
\end{array}\right.
$$

By induction over $\tau \leq \Delta^{k}$ we obtain the desired identification. Again, Figure 1 in the special case when $X=S^{0}$ and $k=1$ might be helpful.

With this identification, starting with a block map $h: X \times \Delta^{k} \rightarrow Y \times \Delta^{k}$ we construct a block map $C_{k}(h): C X \times \Delta^{k} \rightarrow C Y \times \Delta^{k}$ by iterating the construction of the cone map as described in the case $k=0$. The resulting map clearly has the property $C_{k}(h)\left(r \cdot X \times \Delta^{k}\right) \subseteq r \cdot Y \times \Delta^{k}$, and it is our definition of the map $C^{+}(h)$ on $C X \times \Delta^{k}$. It is easy to verify that the maps $T^{\prime}, f^{\prime}$ defined via this procedure define a structure on $V \times \Delta^{k}$ as required.

Proof of Proposition 2.4. The composition res o cone is the identity. It remains to show that the composition cone o res is homotopic to the identity. We start with a structure $(T, f)$ on $V \times \Delta^{k}$, consider its restriction, the structure $(T|, f|)$ on $S(V) \times \Delta^{k}$, and the image $\left(T^{\prime}, f^{\prime}\right)=\operatorname{cone}(T, f)$, a structure on $V \times \Delta^{k}$. We need to find compatible homotopies between $T$ and $T^{\prime}$ and $f$ and $f^{\prime}$ satisfying certain restrictions.

Similarly as in the previous construction we give a general procedure as follows. Suppose $h: C^{+} X \times \Delta^{k} \rightarrow C^{+} Y \times \Delta^{k}$ is a block map such that $h\left(r \cdot X \times \Delta^{k}\right) \subseteq$ $\left(r \cdot Y \times \Delta^{k}\right)$. Consider the restriction block map $h \mid: X \times \Delta^{k} \rightarrow Y \times \Delta^{k}$ and the block map $C_{k}^{+}(h \mid): C^{+} X \times \Delta^{k} \rightarrow C^{+} Y \times \Delta^{k}$ obtained via the iterated cone construction. We give a construction of a homotopy, say $h_{t}$, between $h$ and $C_{k}^{+}(h \mid)$ with the property that each $h_{t}$ is a block map $C^{+} X \times \Delta^{k} \rightarrow C^{+} Y \times \Delta^{k}$ such that $h_{t}\left(r \cdot X \times \Delta^{k}\right) \subseteq\left(r \cdot Y \times \Delta^{k}\right)$. Applying this homotopy to the maps $T, f$ in place of $h$ produces the desired homotopies between $T, f$ and $T^{\prime}, f^{\prime}$.

The case $k=0$. The required homotopy is obtained using the Alexander trick. We can think of the map $h$ as of a perturbation of $C^{+}(h \mid)$ and refer to the part of $C^{+} X$ on which the two maps $h$ and $C^{+}(h \mid)$ do not agree as the domain of the perturbation. Further we consider $C^{+} X=C X \cup C^{1+} X$ and we give a different description of the homotopy on the two pieces. In words, the trick consists of shrinking the domain of the perturbation in $C X$ and of pushing the domain of the perturbation towards $\infty$ in $C^{1+} X$.

Formally, the homotopy $h_{t}$ on $C X$ is parametrized by $t \in[0,1]$ and given by

$$
h_{t}(r x)= \begin{cases}r h(1 x) & \text { for } r \geq t \\ r h(x, r / t) & \text { for } r<t\end{cases}
$$

The homotopy $h_{t}$ on $C^{1+} X$ is parametrized by $[1, \infty]$ and given by

$$
h_{t}(r x)= \begin{cases}r h(1 x) & \text { for } r \leq t \\ r h((1+r-t) x) & \text { for } r \geq t\end{cases}
$$


if $t \in[1, \infty)$ and by $h_{\infty}(r x)=r h(1 x)$. After reparametrizing, using a suitable homeomorphism $[1, \infty] \cong[0,1]$ which maps 1 to 1 and $\infty$ to 0 , we obtain that $h_{0}=C^{+}\left(\left.h\right|_{X}\right)$ and $h_{1}=h$. Moreover, clearly $h_{t}(r \cdot X) \subseteq r \cdot Y$ for all $t \in[0,1]$.

The cases $k>0$. Again, the homotopy is described on the two pieces of the decomposition $C^{+} X \times \Delta^{k}=C X \times \Delta^{k} \cup C^{1+} X \times \Delta^{k}$ differently. On $C^{1+} X \times \Delta^{k}$ it is given by pushing the domain of the perturbation towards $\infty$ just as in the case $k=0$; the formula is left for the reader.

To describe the homotopy on $C X \times \Delta^{k}$, recall that in the definition of $C_{k}(h \mid)$ we iterated the cone construction. Further, in the case $k=0$ the homotopy between $h$ and $C(h \mid)$ was given by the Alexander trick. In analogy, in the case $k>0$ the homotopy between $h$ and $C_{k}(h \mid)$ is given by iterating the Alexander trick.

To start, recall the identification of $C_{k}\left(X \times \Delta^{k}\right)$ with $C X \times \Delta^{k}$ and apply the Alexander trick homotopy to $h$ considered as a block map from $C_{k}\left(X \times \Delta^{k}\right)$ to $C_{k}\left(Y \times \Delta^{k}\right)$. The resulting map, say $h_{1}$, coincides with $C_{k}(h \mid)$ on $C\left(X \times \Delta^{k}\right)$, but is possibly different on the complement, the union of $C\left(C_{k-1}(X \times \tau)\right)$ where $\tau<\Delta^{k}$, $|\tau|=k-1$ (see Figure 1). Note however, that if we consider $C\left(C_{k-1}(X \times \tau)\right.$ ) for each such $\tau$ as the quotient of $C_{k-1}(X \times \tau) \times[0,1]$, then the restriction of $h_{1}$ is the quotient map of the product map of the restriction of $h$ to $C_{k-1}(X \times \tau)$ with the identity on $[0,1]$ (again see Figure 1). Hence, we can perform a homotopy given as the product of the Alexander trick homotopy on $C_{k-1}(X \times \tau)$ and the identity on $[0,1]$. The resulting map now agrees with $C_{k}(h \mid)$ on a larger portion of $C X \times \Delta^{k}$. We proceed inductively until we take care of all the faces of $\Delta^{k}$. Then the resulting map agrees with $C_{k}(h \mid)$ on the whole $C X \times \Delta^{k}$. Again, it is clear that the homotopy $h_{t}$ is such that for all $t$ we have $h_{t}\left(r \cdot X \times \Delta^{k}\right) \subset r \cdot Y \times \Delta^{k}$ for all $r \geq 0$.

Before we discuss continuity of the functor $F$ recall that, since the maps $T, f$ can vary continuously, the set of $k$-simplices of the $\Delta$-groupoid $\widetilde{\mathcal{S}}_{\mathbb{Z}_{2}}^{s}(V)$ (similarly $\left.\widetilde{\mathcal{S}}_{\mathbb{Z}_{2}}^{s}(S(V))\right)$ is endowed with a topology, namely the subspace topology of the product topology of the compact-open topology on the space of self-maps on $V \times \Delta^{k}$. So we have a topological $\Delta$-groupoid, and the associated $\Delta$-set $\widetilde{\mathcal{S}}_{\mathbb{Z}_{2}}^{s}(V)$ becomes a $\Delta$-space.

Lemma 2.7. The geometric realization of $\widetilde{\mathcal{S}}_{\mathbb{Z}_{2}}^{s}(V)$ as a $\Delta$-space is homotopy equivalent to the geometric realization of the same object as a $\Delta$-set.

Proof. To see this we resolve the $\Delta$-space version of $\widetilde{\mathcal{S}}_{\mathbb{Z}_{2}}^{s}(V)$ by taking the singular simplicial set of the space of $k$-simplices for each $k \in \mathbb{N}$. We obtain a combined simplicial- $\Delta$-set, which has its geometric realization homotopy equivalent to the geometric realization of the $\Delta$-space. We can 'change the order' of realization and realize first spaces maps $\left(\Delta^{k}, \widetilde{\mathcal{S}}_{\mathbb{Z}_{2}}^{s}(V)\right)$ for each $k \in \mathbb{N}$ as $\Delta$-sets and then realize the resulting simplicial set. The lemma will be proved if we show that all degeneracy and face maps between maps $\left(\Delta^{k}, \widetilde{\mathcal{S}}_{\mathbb{Z}_{2}}^{s}(V)\right)$ and maps $\left(\Delta^{k+1}, \widetilde{\mathcal{S}}_{\mathbb{Z}_{2}}^{s}(V)\right)$ induce homotopy equivalences. This can be verified using the Kan property of $\widetilde{\mathcal{S}}_{\mathbb{Z}_{2}}^{s}(V)$.

Now we show that the functor $F$ is a continuous functor in the sense of Definition 3.1. First, note that one can define a continuous functor from $\mathcal{J}$ to topological $\Delta$ groupoids or to $\Delta$-spaces to be a functor for which for all $k$ the evaluation maps

$$
\mathrm{ev}_{k}: \operatorname{mor}(V, W) \times E(V)_{k} \rightarrow E(W)_{k}
$$


are continuous. Given such a continuous functor from $E: \mathcal{J} \rightarrow$ top $-\Delta$-groupoids ${ }_{*}$, composing with the functor $E \mapsto E^{\sharp}$ and taking the geometric realization of $E^{\sharp}$ we obtain a continuous functor to Spaces $_{*}$.

Theorem 2.8. The functor $F: \mathcal{J} \rightarrow$ Spaces $_{*}$ is a continuous functor.

Proof. In view of the previous remarks it is enough to show that the functor $F: V \mapsto$ $\widetilde{\mathcal{S}}_{\mathbb{Z}_{2}}^{s}(V)$ is continuous. That means we need to show that the evaluation map

$$
\mathrm{ev}_{k}: \operatorname{mor}(V, W) \times F(V)_{k} \rightarrow F(W)_{k}
$$

is continuous for each $k$.

If $\operatorname{dim}(V)=\operatorname{dim}(W)$, then $\mathrm{ev}_{k}$ is given by $(\xi,(T, f)) \mapsto\left(\xi \circ T \circ \xi^{-1}, \xi \circ f \circ \xi^{-1}\right)$ which clearly gives continuity.

For the case when $\operatorname{dim}(V)<\operatorname{dim}(W)$ we work locally and reduce to the above case as follows. Choose $\xi: V \rightarrow W$. We prove that the evaluation map is continuous on some neighborhood $V_{\xi}$ of $\xi$. There is a map $\operatorname{mor}(W, W) \rightarrow \operatorname{mor}(V, W)$ given by $\zeta \mapsto \zeta \circ \xi$, which is a bundle map. Therefore there is a neighborhood $V_{\xi} \subset \operatorname{mor}(V, W)$ of $\xi$ over which we have a section $s$ of this bundle. Then the evaluation map $\mathrm{ev}_{k}$ can be factored as

$$
V_{\xi} \times F(V)_{k} \stackrel{s \times F(\xi)_{k}}{\longrightarrow} \operatorname{mor}(W, W) \times F(W)_{k} \stackrel{\mathrm{ev}_{k}}{\longrightarrow} F(W)_{k} .
$$

The first map is continuous because by the assumption both of its factors are continuous and the second one is the previous case.

\section{Orthogonal Calculus}

Our method of study of the functor $F$ defined in the previous section is the orthogonal calculus of functors. In this section we review some of the terms and tools of orthogonal calculus which we use in this paper. It is intended as a short survey which describes the context in which we work. For the full account we refer the reader to the paper of Weiss We in which orthogonal calculus was developed.

We recall some notation. By $\mathcal{J}$ we denote the category of finite-dimensional real vector spaces with (positive definite) inner product. Morphisms are linear maps preserving inner product, in particular, they are linear inclusions. The objects of $\mathcal{J}$ are usually denoted by the letters $U, V, W$. Morphism sets $\operatorname{mor}(V, W)$ are Stiefel manifolds and so have a topology. By $\iota$ we will denote the inclusion of $V$ into $V \oplus U$ for any objects $V, U$ in $\mathcal{J}$. In special cases, by $\iota_{1}$ we will denote the inclusion of $V \oplus \mathbb{R}$ into $V \oplus \mathbb{R} \oplus \mathbb{R}$ where $\mathbb{R}$ is mapped isomorphically onto $\mathbb{R} \oplus\{0\}$ in $\mathbb{R} \oplus \mathbb{R}$ and by $\iota_{2}$ the inclusion where $\mathbb{R}$ is mapped onto $\{0\} \oplus \mathbb{R}$ in $\mathbb{R} \oplus \mathbb{R}$. For $U \in \operatorname{Ob}(\mathcal{J}), U^{c}$ denotes the one-point compactification of $U$. Furthermore $\mathbb{R}_{+}=\{u \in \mathbb{R} \mid u \geq 0\}$ and $\mathbb{R}_{+}^{c}=\mathbb{R}_{+} \cup\{\infty\}$. We also write $k \cdot U$ for $\mathbb{R}^{k} \otimes U$.

The orthogonal calculus works for functors $E: \mathcal{J} \rightarrow$ Spaces $_{*}$ satisfying the following axiom.

Definition 3.1 (We $)$. A functor $E: \mathcal{J} \rightarrow$ Spaces $_{*}$ is called continuous if for each $V, W \in \mathrm{Ob}(\mathcal{J})$ the evaluation map

$$
\text { ev }: \operatorname{mor}(V, W) \times E(V) \rightarrow E(W)
$$

is continuous. 
We have shown in Theorem 2.8 that the functor $F$ satisfies this axiom. There are many other functors of this type. For example, the functor $V \mapsto \mathrm{BO}(V)$, where $\mathrm{BO}(V)$ is the classifying space of the orthogonal group $\mathrm{O}(V)$, has been studied in $\mathrm{Ar}$. Other examples come up in the study of automorphisms of manifolds; see papers $\mathrm{We}, \mathrm{WW}$.

We have already presented the basic philosophy of orthogonal calculus in the Introduction. Recall that a continuous functor $E: \mathcal{J} \rightarrow$ Spaces $_{*}$ is studied by means of its Taylor tower which is a tower of functors

$$
\cdots \rightarrow T_{k} E \rightarrow T_{k-1} E \rightarrow \cdots \rightarrow T_{0} E,
$$

where the functor $T_{k} E$ is the $k$-th polynomial approximation of $E$. The tower should be thought of as the expansion at infinity. There are two aspects of the Taylor tower which one needs to understand. First, one needs to find out whether the Taylor tower converges to $E$, that means to estimate the connectivity of the canonical maps $E(V) \rightarrow T_{k} E(V)$. Second, one needs to determine the differences, hofiber $_{*}\left(T_{k} E(V) \rightarrow T_{k-1} E(V)\right)$, between the stages of the tower.

Both issues can be investigated using the derivatives in orthogonal calculus. These arise as follows. Given a continuous functor $E: \mathcal{J} \rightarrow$ Spaces $_{*}$ there is defined for each $k \in \mathbb{N}$ its $k$-th derivative functor $E^{(k)}$ which is again a continuous functor from $\mathcal{J}$ to Spaces $_{*}$ with the additional structure of a coordinate-free spectrum of multiplicity $k$. That means there are maps

$$
\sigma_{k}:(k \cdot U)^{c} \wedge E^{(k)}(V) \rightarrow E^{(k)}(V \oplus U) .
$$

On objects, $E^{(k+1)}$ is defined inductively as

$$
E^{(k+1)}(V)=\operatorname{hofiber}_{*}\left(\tilde{\sigma}_{k}: E^{(k)}(V) \rightarrow \Omega^{k} E^{(k)}(V \oplus \mathbb{R})\right),
$$

where $\tilde{\sigma}_{k}$ is the adjoint of $\sigma_{k}$. For the definition on morphisms, take $\xi \in \operatorname{mor}(V, W)$ and define $\xi^{\prime} \in \operatorname{mor}(V \oplus \mathbb{R}, W \oplus \mathbb{R})$ by $\xi^{\prime}(v, x)=(\xi(v), x)$. The pair $\left(\xi, \xi^{\prime}\right)$ then defines the map $E^{(k+1)}(\xi)$ between the homotopy fibers $E^{(k+1)}(V) \rightarrow E^{(k+1)}(W)$. In Construction 3.2 we confine ourselves to a description of the maps $\sigma_{k}$ for $k=1$, as we will not need the maps $\sigma_{k}$ for $k>1$.

Construction 3.2. The values $E^{(1)}(V)$ are defined as homotopy fibers of the stabilization maps $E(\iota): E(V) \rightarrow E(V \oplus \mathbb{R})$, where $\iota: V \rightarrow V \oplus \mathbb{R}$ is the linear inclusion. A point in $E^{(1)}(V)$ is described as a pair $(x, \lambda)$, where $x \in E(V)$ and $\lambda: \mathbb{R}_{+}^{c} \rightarrow E(V \oplus \mathbb{R})$ satisfies $\lambda(0)=E(\iota)(x)$ and $\lambda(\infty)=*$.

We give a description of the adjoints of the maps $\sigma_{1}$, that means the maps

$$
\tilde{\sigma}_{1}: E^{(1)}(V) \rightarrow \Omega^{U} E^{(1)}(V \oplus U) .
$$

Given $(x, \lambda) \in E^{(1)}(V)$ we describe the image $\left(x^{\prime}, \lambda^{\prime}\right):=\tilde{\sigma}(x, \lambda) \in \Omega^{U} E^{(1)}(V \oplus U)$, where

$$
\begin{gathered}
x^{\prime}: U^{c} \rightarrow E(V \oplus U), \\
\lambda^{\prime}:\left(U \oplus \mathbb{R}_{+}\right)^{c} \rightarrow E(V \oplus U \oplus \mathbb{R}),
\end{gathered}
$$

such that $x^{\prime}(\infty)=*,\left.\lambda^{\prime}\right|_{(U \oplus\{0\})^{c}}=E(\iota)\left(x^{\prime}\right)$ and $\lambda^{\prime}(\infty)=*$.

Each vector $u \in U$, such that $\|u\|=1$, defines a morphism $\xi_{u}: V \oplus \mathbb{R} \rightarrow V \oplus U$ in $\mathcal{J}$ by $\xi_{u}:(v, 1) \mapsto(v, u)$.

Similarly, each vector $w \in U \oplus \mathbb{R}_{+}$, such that $\|w\|=1$, defines a morphism $\zeta_{w}: V \oplus \mathbb{R} \rightarrow V \oplus U \oplus \mathbb{R}$ in $\mathcal{J}$ by $\zeta_{w}:(v, 1) \mapsto(v, w)$. 
All such unit vectors $u \in U, w \in U \oplus \mathbb{R}_{+}$define rays $\langle u\rangle$ and $\langle w\rangle$ from 0 through $u$ and $w$, which cover $U$ and $U \oplus \mathbb{R}_{+}$, respectively. We define $x^{\prime}, \lambda^{\prime}$ on the closures $\langle u\rangle^{c} \subseteq U^{c},\langle w\rangle^{c} \subseteq\left(U \oplus \mathbb{R}_{+}\right)^{c}$ as the compositions

$$
\begin{gathered}
x^{\prime}:\langle u\rangle^{c} \stackrel{\cong}{\longrightarrow} \mathbb{R}_{+}^{c} \stackrel{\lambda}{\rightarrow} E(V \oplus \mathbb{R}) \stackrel{E\left(\xi_{u}\right)}{\longrightarrow} E(V \oplus U), \\
\lambda^{\prime}:\langle w\rangle^{c} \stackrel{\cong}{\longrightarrow} \mathbb{R}_{+}^{c} \stackrel{\lambda}{\longrightarrow} E(V \oplus \mathbb{R}) \stackrel{E\left(\zeta_{w}\right)}{\longrightarrow} E(V \oplus U \oplus \mathbb{R}) .
\end{gathered}
$$

Here the fact that the functor $E$ is continuous is used. It guarantees that the maps $x^{\prime}, \lambda^{\prime}$ are continuous.

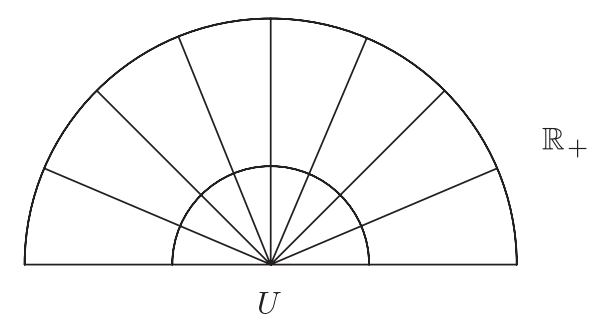

FiguRE 2. Schematic picture of the map $\tilde{\sigma}_{1}$

Figure 2 describes the map $\tilde{\sigma}_{1}$. Lines inside the half-disk represent the rays $\langle w\rangle$, and the half-circle represents the unit hemisphere inside $U \oplus \mathbb{R}_{+}$. The morphism $\zeta_{w} \in \operatorname{mor}(V \oplus U, V \oplus U \oplus \mathbb{R})$ which is used to define $\lambda^{\prime}$ on each ray $\langle w\rangle$ is determined by the point on the unit hemisphere which the ray $\langle w\rangle$ intersects.

Now we turn our attention to the convergence question. We will not address this issue in full generality, that means we will not discuss a notion of an analytic functor analogous to such a notion in Go. We will only consider the case when a functor $E$ is polynomial of some degree $\leq k$. In this case the Taylor tower 'stops' at the $k$-th stage, that means the canonical maps $E(V) \rightarrow T_{k} E(V)$ are homotopy equivalences and thus the $k$-th stage $T_{k} E$ gives us complete homotopy theoretical information about the functor $E$. Polynomial functors are defined as follows.

Definition 3.3. A continuous functor $E: \mathcal{J} \rightarrow$ Spaces $_{*}$ is called polynomial of degree $\leq k$ if for every $V \in \mathcal{J}$ the following canonical map is a homotopy equivalence:

$$
E(V) \longrightarrow \operatorname{holim}_{0 \neq U \subseteq \mathbb{R}^{k+1}} E(V \oplus U) .
$$

The homotopy limit is taken over the topological poset of all non-zero vector subspaces of $\mathbb{R}^{k+1}$ (see [We, section 5]).

In general, for a functor $E: \mathcal{C} \rightarrow$ Spaces $_{*}$ from a small category $\mathcal{C}$, the homotopy limit of $E$ over $\mathcal{C}$ can be defined as the total space of a cosimplicial space given by

$$
\mathbf{l} \mapsto \prod_{\sigma: 1 \rightarrow \mathcal{C}} E(\sigma(l)) ;
$$

see $\mathrm{BK}$. If $\mathcal{C}$ is a topological category, this affects the definition of the homotopy limit. In our case, when $\mathcal{C}$ is $\left\{0 \neq U \subseteq \mathbb{R}^{k+1}\right\}$ with objects non-zero vector subspaces of $\mathbb{R}^{k+1}$ and one morphism for every inclusion $U \subseteq V \subseteq \mathbb{R}^{k+1}$, the sets $\{\sigma: \mathbf{l} \rightarrow \mathcal{C}\}$ become a disjoint union of Stiefel manifolds. Then the disjoint union of spaces $E(\sigma(l))$ becomes a bundle over $\{\sigma: \mathbf{l} \rightarrow \mathcal{C}\}$. The space of $l$-cosimplices of 
the cosimplicial space which defines the homotopy limit then becomes the space of sections of this bundle; see [We, sections 4,5] for more details.

The property above is an extrapolation property. Note that the value of the functor $E$ at $V$ is determined by the values of $E$ at vector spaces between $V$ and $V \oplus \mathbb{R}^{k+1}$ which are strictly greater than $V$. For more details see [We, section 5].

The derivatives are related to the polynomial functors via the following proposition [We, Proposition 5.3].

Proposition 3.4. For any $V \in \mathcal{J}$ the value $E^{(k+1)}(V)$ is homotopy equivalent to the homotopy fiber of the canonical map

$$
E(V) \rightarrow \operatorname{holim}_{0 \neq U \subseteq \mathbb{R}^{k+1}} E(V \oplus U)
$$

over the base point of the target.

Thus if the functor $E$ is polynomial of degree $\leq k$, then the $(k+1)$-th derivative functor $E^{(k+1)}$ vanishes. If the target of the map from Proposition 3.4 is pathconnected for all $V$, then the converse is also true. If the target is not pathconnected, then we also have to investigate the homotopy fibers over other points than the base point. This leads to a necessary and sufficient condition for $E$ to be polynomial of degree $\leq k$ which we state in Theorem 3.6 in the case $k=1$. It is due to Weiss (but did not appear in We ), and we are grateful to him for showing it to us. Before we state it we need to define a modification of the map $\tilde{\sigma}_{1}$ presented in Construction 3.5.

We also need some new notation because we will use compactifications of Euclidean spaces other than the one-point compactification. For $V \in \operatorname{Ob}(\mathcal{J})$ by $\bar{V}$ we denote the compactification of $V$ by the sphere $\partial \bar{V}=\{\bar{w} \mid w \in V,\|w\|=1\}$ at infinity. In special cases, $\overline{\mathbb{R}}$ is the 0 -sphere compactification of $\mathbb{R}$, with the points at infinity $\pm \overline{1}$, and $\overline{\mathbb{R} \oplus \mathbb{R}}$ is the 1 -sphere compactification of $\mathbb{R} \oplus \mathbb{R}$ with points at infinity $\partial \overline{\mathbb{R} \oplus \mathbb{R}}=\left\{\bar{w} \mid w \in \mathbb{R}^{2},\|w\|=1\right\}$. Here we also distinguish $\partial_{ \pm} \overline{\mathbb{R} \oplus \mathbb{R}}=\left\{\bar{w} \mid w=\left(w_{1}, w_{2}\right), \pm w_{2} \geq 0\right\}$. By $\overline{\mathbb{R} \oplus \mathbb{R}_{+}}$we denote the 1-disk compactification of $\mathbb{R} \oplus \mathbb{R}_{+}$, points at infinity are $\left\{\bar{w} \mid w \in \mathbb{R} \oplus \mathbb{R}_{+},\|w\|=1\right\}$.

Construction 3.5. Denote $E^{(1)}(V, y)=\operatorname{hofiber}_{y}(E(V) \rightarrow E(V \oplus \mathbb{R}))$, where hofiber $_{y}$ means the homotopy fiber taken over some point $y \in E(V \oplus \mathbb{R})$.

Let $X_{y}$ denote the space of pairs of maps $\left(x^{\prime}, \lambda^{\prime}\right)$ where

$$
\begin{gathered}
x^{\prime}: \overline{\mathbb{R}} \rightarrow E(V \oplus \mathbb{R}), \\
\lambda^{\prime}: \overline{\mathbb{R} \oplus \mathbb{R}_{+}} \rightarrow E\left(V \oplus \mathbb{R}^{2}\right),
\end{gathered}
$$

such that $x^{\prime}( \pm \overline{1})= \pm y$, where $-y$ denotes the image of the point $y$ under the self-map of $E(V \oplus \mathbb{R})$ induced by the reflection of $\mathbb{R}$. Furthermore we require that $\left.\lambda^{\prime}\right|_{\overline{\mathbb{R}} \oplus\{0\}}=E\left(\iota_{1}\right)\left(x^{\prime}\right)$ and $\lambda^{\prime}(\bar{w})=E\left(\zeta_{w}\right)(y)$, where $\zeta_{w}$ is as in Construction 3.2

Note that in the case $y=*$ the space $X_{y}$ is homotopy equivalent to the loop space $\Omega E^{(1)}(V \oplus \mathbb{R})$. If $y \neq *$ the space $X_{y}$ can be thought of as a certain space of paths in $E^{(1)}\left(V \oplus \mathbb{R}, y^{\prime}\right)$, where $y^{\prime}=E\left(\zeta_{w^{\prime}}\right)(y)$ and where $w^{\prime}=(0,0,1)$. This is depicted in Figure 3 .

The map $\tilde{\sigma}_{1}: E^{(1)}(V, y) \rightarrow X_{y}$ is defined similarly as the map $\tilde{\sigma}_{1}$ in Construction 3.2. For $(x, \lambda) \in E^{(1)}(V, y)$ we define $\left(x^{\prime}, \lambda^{\prime}\right)=\tilde{\sigma}_{1}(x, \lambda) \in X_{y}$ as follows. Given unit vectors $u \in \mathbb{R}, w \in \mathbb{R} \oplus \mathbb{R}_{+}$we now have rays $\langle 0, \bar{u}\rangle,\langle 0, \bar{w}\rangle$ from 0 to $\bar{u}$ or $\bar{w}$. 


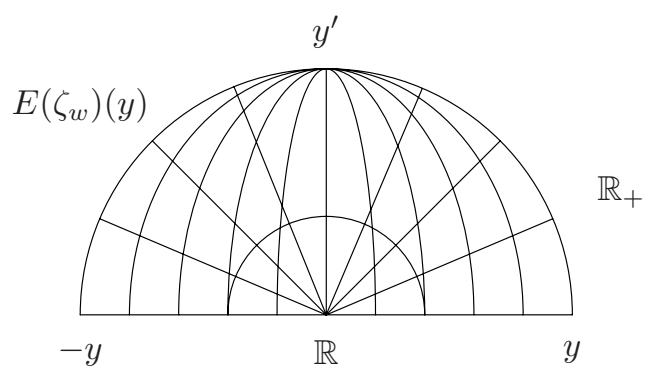

FiguRE 3. Schematic picture of $\lambda^{\prime}$ and of the map $\tilde{\sigma}_{1}$.

These rays cover $\overline{\mathbb{R}}, \overline{\mathbb{R} \oplus \mathbb{R}_{+}}$respectively. Define

$$
\begin{aligned}
x^{\prime} & \langle 0, \bar{u}\rangle \stackrel{\cong}{\longrightarrow} \mathbb{R}_{+}^{c} \stackrel{\lambda}{\rightarrow} E(V \oplus \mathbb{R}) \stackrel{E\left(\xi_{u}\right)}{\longrightarrow} E(V \oplus \mathbb{R}), \\
\lambda^{\prime}:\langle 0, \bar{w}\rangle \cong & \stackrel{\cong}{\longrightarrow} \mathbb{R}_{+}^{c} \stackrel{\lambda}{\longrightarrow} E(V \oplus \mathbb{R}) \stackrel{E\left(\zeta_{w}\right)}{\longrightarrow} E\left(V \oplus \mathbb{R}^{2}\right) .
\end{aligned}
$$

If $y=*$, then the map $\tilde{\sigma}_{1}$ obviously coincides with the map $\tilde{\sigma}_{1}$ from Construction 3.2, see Figure 3 again.

Now we are ready to state the promised necessary and sufficient condition.

Theorem 3.6. A continuous functor $E: \mathcal{J} \rightarrow$ Spaces $_{*}$ is polynomial of degree $\leq 1$ if and only if for all $V \in \mathcal{J}$ and for all $y \in E(V \oplus \mathbb{R})$ the map $\tilde{\sigma}_{1}: E^{(1)}(V, y) \rightarrow X_{y}$ defined in Construction 3.5 is a homotopy equivalence.

Proof. We sketch the proof. We need to show that the map

$$
E(V) \rightarrow \operatorname{holim}_{0 \neq U \subset \mathbb{R}^{2}} E(V \oplus U)
$$

is a homotopy equivalence if and only if for all choices of $V$ and $y$ the map $\tilde{\sigma}_{1}$ is also a homotopy equivalence.

Consider the map $E(V) \rightarrow E(V \oplus \mathbb{R})$ induced by $\iota: V \rightarrow V \oplus \mathbb{R}$ and the map holim $_{0 \neq U \subset \mathbb{R}^{2}} E(V \oplus U) \rightarrow E(V \oplus \mathbb{R})$ obtained by the restriction via $\iota_{1}: V \oplus \mathbb{R} \rightarrow V \oplus \mathbb{R}^{2}$. For $y \in E(V \oplus \mathbb{R})$ consider the induced map, say $\tilde{\sigma}_{y}$, between the homotopy fibers over $y$ of both maps. The second map is a fibration, so the target homotopy fiber can be replaced by the fiber over $y$. In Lemma 3.7 below we show that the space $X_{y}$ is homotopy equivalent to this fiber. The map $\tilde{\sigma}_{1}$ is such that it is homotopic to the composition of the map $\tilde{\sigma}_{y}$, a suitable homotopy equivalence from the homotopy fiber to the fiber over $y$ of the map $\operatorname{holim}_{0 \neq U \subset \mathbb{R}^{2}} E(V \oplus U) \rightarrow E(V \oplus \mathbb{R})$ and the identification of this fiber with the space $X_{y}$ given in Lemma 3.7 (this should be clear after reading the proof of Lemma 3.7 below). Now the map (3.3) is a homotopy equivalence if and only if for all choices of $y$ the map $\tilde{\sigma}_{y}$ is also a homotopy equivalence.

Lemma 3.7. The space $X_{y}$ is homotopy equivalent to the fiber of the map

$$
\operatorname{holim}_{0 \neq U \subset \mathbb{R}^{2}} E(V \oplus U) \rightarrow E(V \oplus \mathbb{R})
$$

over the point $y$. 
Proof. As a topological space the category $\left\{0 \neq U \subseteq \mathbb{R}^{2}\right\}$ is $\mathbb{R} P\left(\mathbb{R}^{2}\right) \amalg\left\{\mathbb{R}^{2}\right\}$, with one morphism (an inclusion, denoted by $\iota_{U}$ ) for every point of $U \in \mathbb{R} P\left(\mathbb{R}^{2}\right)$ to $\mathbb{R}^{2}$. We will also denote by the same $\iota_{U}$ the inclusion of $V \oplus U$ into $V \oplus \mathbb{R}^{2}$.

According to the definition the homotopy limit of the functor $E(V \oplus-)$ over this category is given by a pair of maps

$$
\begin{array}{ll}
\lambda_{0}: & \mathbb{R} P\left(\mathbb{R}^{2}\right) \rightarrow E_{0}, \\
\lambda_{1}: & \mathbb{R} P\left(\mathbb{R}^{2}\right) \times \Delta^{1} \rightarrow E\left(V \oplus \mathbb{R}^{2}\right),
\end{array}
$$

where $E_{0}$ is a bundle over the circle $\mathbb{R} P\left(\mathbb{R}^{2}\right)$ with the fiber over $U$ the space $E(V \oplus U)$ and such that $\lambda_{0}$ is a section of this bundle, $\lambda_{1}(U, 0)=E\left(\iota_{U}\right)\left(\lambda_{0}(U)\right)$ and $\lambda_{1}(U, 1)=$ $\lambda_{1}\left(U^{\prime}, 1\right)$ for every $U, U^{\prime}$ in $\mathbb{R} P\left(\mathbb{R}^{2}\right)$. In a more condensed form we can say that the source of the map $\lambda_{1}$ is defined just on the cone over the circle $\mathbb{R} P\left(\mathbb{R}^{2}\right)$. The projection to $E(V \oplus \mathbb{R})$ is given by $\lambda_{0}(\mathbb{R} \oplus\{0\})$, therefore taking the fiber over $y \in E(V \oplus \mathbb{R})$ means to require in addition that $\lambda_{0}(\mathbb{R} \oplus\{0\})=y$.

Note that we can think of $E_{0}$ as a subbundle of the trivial bundle over $\mathbb{R} P\left(\mathbb{R}^{2}\right)$ with the fiber $E\left(V \oplus \mathbb{R}^{2}\right)$. The compatibility conditions between $\lambda_{0}$ and $\lambda_{1}$ express the fact that the two sections of the two bundles commute with the embedding of the subbundle. The identification of the fiber over $y$ with the space $X_{y}$ is obtained via a sequence of reparametrizations of the maps $\lambda_{0}, \lambda_{1}$ which correspond to certain isotopies of the embedding of $E_{0}$ into $E\left(V \oplus \mathbb{R}^{2}\right) \times \mathbb{R} P\left(\mathbb{R}^{2}\right)$ as follows. (The reader might find it useful to draw some pictures of the situation similar to Figure 3.)

First, recall the 1-sphere compactification $\overline{\mathbb{R} \oplus \mathbb{R}}$ of $\mathbb{R} \oplus \mathbb{R}$ with points at infinity $\partial \overline{\mathbb{R} \oplus \mathbb{R}}=\left\{\bar{w} \mid w \in \mathbb{R}^{2},\|w\|=1\right\}$ and redefine the fiber over $y$ as the space of maps

$$
\begin{array}{ll}
\lambda_{0}: & \partial \overline{\mathbb{R} \oplus \mathbb{R}} \rightarrow E_{0}, \\
\lambda_{1} & : \quad \overline{\mathbb{R} \oplus \mathbb{R}} \rightarrow E\left(V \oplus \mathbb{R}^{2}\right),
\end{array}
$$

such that for $w=\left(w_{1}, w_{2}\right) \in \mathbb{R} \oplus \mathbb{R}$ with $\|w\|=1$ we have $\lambda_{0}(\bar{w}) \in E(V \oplus\langle w\rangle)$ if $w_{2} \leq 0$ and $\lambda_{0}(\bar{w})=y \in E(V \oplus \mathbb{R} \oplus\{0\})$ if $w_{2} \geq 0$, and $\lambda_{1}(\bar{w})=E\left(\iota_{\langle w\rangle}\right)\left(\lambda_{0}(\bar{w})\right)$ if $w_{2} \leq 0$ and $\lambda_{1}(\bar{w})=E\left(\iota_{1}\right)(y)$ if $w_{2} \geq 0$. Here $\langle w\rangle$ denotes the vector subspace of $\mathbb{R}^{2}$ generated by $w$.

By 'rotating' the fibers of $E_{0}$ inside $E\left(V \oplus \mathbb{R}^{2}\right)$, we redefine our space again as the space of maps $\left(\lambda_{0}, \lambda_{1}\right)$ as above, but now satisfying $\lambda_{0}(\bar{w}) \in E(V \oplus \mathbb{R})$ if $w_{2} \leq 0$ and $\lambda_{0}(\bar{w})=E\left(\xi_{w}\right)(y) \in E(V \oplus\langle w\rangle)$ if $w_{2} \geq 0$, and $\lambda_{1}(\bar{w})=E\left(\iota_{1}\right)\left(\lambda_{0}(\bar{w})\right)$ if $w_{2} \leq 0$ and $\lambda_{1}(\bar{w})=E\left(\iota_{\langle w\rangle}\right)\left(\lambda_{0}(\bar{w})\right)=E\left(\zeta_{w}\right)(y)$ if $w_{2} \geq 0$, where $\xi_{w}: V \oplus \mathbb{R} \rightarrow V \oplus\langle w\rangle$ and $\zeta_{w}: V \oplus \mathbb{R} \rightarrow V \oplus \mathbb{R}^{2}$ are as in Construction 3.2

In the next step consider $\partial_{-} \overline{\mathbb{R} \oplus \mathbb{R}}$ and redefine the fiber over $y$ as the space of maps

$$
\begin{array}{lll}
\lambda_{0}: & \partial_{-} \overline{\mathbb{R} \oplus \mathbb{R}} \rightarrow E(V \oplus \mathbb{R}), \\
\lambda_{1} & : & \overline{\mathbb{R} \oplus \mathbb{R}} \rightarrow E\left(V \oplus \mathbb{R}^{2}\right),
\end{array}
$$

such that for $w=\left(w_{1}, w_{2}\right) \in \mathbb{R} \oplus \mathbb{R}$ with $\|w\|=1$ we have $\lambda_{0}(\overline{( \pm 1,0)})= \pm y$, and $\lambda_{1}(\bar{w})=E\left(\iota_{1}\right)\left(\lambda_{0}(\bar{w})\right)$ if $w_{2} \leq 0$ and $\lambda_{1}(\bar{w})=E\left(\zeta_{w}\right)(y)$.

Finally choose a homeomorphism $\overline{\mathbb{R} \oplus \mathbb{R}} \rightarrow \overline{\mathbb{R} \oplus \mathbb{R}_{+}}$such that its restriction to $\partial_{-} \overline{\mathbb{R} \oplus \mathbb{R}}$ is the projection onto $\overline{\mathbb{R}} \oplus\{0\}$ and the restriction to $\partial_{+} \overline{\mathbb{R} \oplus \mathbb{R}}$ is the identity. The resulting space of maps is the desired $X_{y}$.

Remark 3.8. Note that if we want to use Theorem 3.6 to prove that a certain functor $E$ is polynomial of degree $\leq 1$, there are two possibilities for each $y \in E(V \oplus \mathbb{R})$; the 
spaces $E^{(1)}(V, y), X_{y}$ might be either empty or not. So the proof splits into two parts. Namely,

(1) show that $E^{(1)}(V, y)$ is empty if and only if $X_{y}$ is empty, and

(2) if $E^{(1)}(V, y)$ is not empty, show that $\tilde{\sigma}_{1}: E^{(1)}(V, y) \rightarrow X_{y}$ is a homotopy equivalence.

This finishes the discussion of polynomial functors. Now we turn to the Taylor tower. We have already mentioned that for a continuous functor $E$ the functors $T_{k} E$ are its polynomial approximations. This is the content of Theorem 6.3 of We which in a slightly weaker form reads as:

Theorem 3.9. Let $E: \mathcal{J} \rightarrow$ Spaces $_{*}$ be a continuous functor. Then for $k \geq 0$ there exists a functor $T_{k} E: \mathcal{J} \rightarrow$ Spaces $_{*}$ and a natural transformation $\eta_{k}: E \rightarrow T_{k} E$ such that:

(1) $T_{k} E$ is polynomial of degree $\leq k$,

(2) if E already is polynomial of degree $\leq k$, then the map

$$
\eta_{k}: E(V) \rightarrow T_{k} E(V)
$$

is a homotopy equivalence for all $V$.

The formula for $T_{k} E$ is given in the proof of Theorem 6.3 in [We. For example, if $k=0$ we have for all $V \in \mathrm{Ob}(\mathcal{J})$

$$
T_{0} E(V)=E\left(V \oplus \mathbb{R}^{\infty}\right)=\underset{m \in \mathbb{N}}{\operatorname{hocolim}} E\left(V \oplus \mathbb{R}^{m}\right) .
$$

Now we address the issue of how to determine the layers of the Taylor tower. They are determined by the $k$-th derivative spectrum $\Theta E^{(k)}$ which is the 'ordinary' spectrum associated to the coordinate-free spectrum given by the $k$-th derivative functor $E^{(k)}$. Namely $\left(\boldsymbol{\Theta} E^{k}\right)_{k m}=E^{(k)}\left(\mathbb{R}^{m}\right)$ and the structure maps are specializations:

$$
\sigma_{k}:\left(\mathbb{R}^{k}\right)^{c} \wedge\left(\boldsymbol{\Theta} E^{(k)}\right)_{k m} \rightarrow\left(\boldsymbol{\Theta} E^{(k)}\right)_{k(m+1)} .
$$

As we have mentioned before the tower is the expansion at infinity, and therefore the spectrum $\Theta E^{(k)}$ should be thought as the $k$-th derivative of $E$ at infinity.

We have also already indicated that there is an action of the orthogonal group $O(k)$ on the $k$-th derivative spectrum associated to $E^{(k)}$. The relevant statement in We] is Proposition 3.1 and, simplified, it reads as:

Proposition 3.10. There exists a unique family $\left\{\alpha_{V}\right\}$ of left actions

$$
\alpha_{V}: O(k) \times E^{(k)}(V) \rightarrow E^{(k)}(V)
$$

which makes the maps

$$
\sigma_{k}:(k \cdot U)^{c} \wedge E^{(k)}(V) \rightarrow E^{(k)}(V \oplus U)
$$

into $O(k)$-maps. Here $O(k)$ acts diagonally on the domain of the map $\sigma_{k}$; it acts on $(k \cdot U)=\mathbb{R}^{k} \otimes U$ because it acts on $\mathbb{R}^{k}$.

As we have said before, the polynomial approximations $T_{k} E$ fit together to form the Taylor tower of $E$ which is the diagram (3.1). The natural transformation $T_{k} E \rightarrow T_{k-1} E$ is essentially given as the $(k-1)$-st polynomial approximation of $T_{k} E$. The usefulness of the Taylor tower follows from the fact that the differences between its stages, hofiber $_{*}\left(T_{k} E(V) \rightarrow T_{k-1} E(V)\right)$, which are called the layers of 
the tower, can be described using the derivatives. The formula is given in Theorem 9.1 of We. It states:

Theorem 3.11. For a continuous functor $E: \mathcal{J} \rightarrow$ Spaces $_{*}$, any $k>0$ and any $V \in \mathcal{J}$, there is the following homotopy fibration sequence:

$$
\Omega^{\infty}\left[\left((k \cdot V)^{c} \wedge \Theta E^{(k)}\right)_{h O(k)}\right] \rightarrow T_{k} E(V) \stackrel{r_{k}}{\longrightarrow} T_{k-1} E(V),
$$

where the subscript $h O(k)$ denotes the homotopy orbit spectrum. The group $O(k)$ acts diagonally on the smash product, it acts on $\Theta E^{(k)}$ by Proposition 3.10 and it acts on $k \cdot V=\mathbb{R}^{k} \otimes V$ because it acts on $\mathbb{R}^{k}$.

Once we have established the convergence question and we know the layers of the Taylor tower, we can use the Taylor tower to obtain some information about the functor $E$. One way to do this is suggested in the Introduction for our functor $F$. The input for the Taylor tower of $F$ is given in Theorems 5.1, and 5.11 For examples on how the Taylor tower can be used to give information about other functors see [We, section 10].

Remark 3.12. The functor $F$ defined in section 2 is a continuous functor $\mathcal{J} \rightarrow$

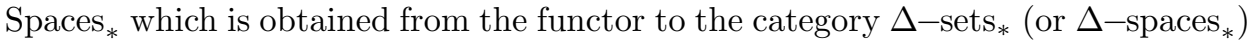
by the geometric realization. Although we do not generalize the machinery of orthogonal calculus to this setting, we often use constructions in the category $\Delta$-sets $*$ (or $\Delta$ - spaces $_{*}$ ), for example the construction of the homotopy fiber of a map or other constructions in Construction 5.3 of section 5. After geometric realization these constructions always commute (at least up to homotopy) with the corresponding constructions in the category Spaces $_{*}$ which justifies their use.

\section{The FIRst DeRIVATIVE FUnCTOR $F^{(1)}$}

Now we start to apply the machinery of orthogonal calculus to study the functor $F$ defined in section 2. The aim of this section is to identify the homotopy type of the spaces $F^{(1)}(V, y)$. This is done in Proposition 4.4, where we give a criterion for the space $F^{(1)}(V, y)$ to be empty and we show that when it is not empty it is homotopy equivalent to a certain space $\mathbf{L} \mathbf{N}_{n}\left(\phi_{V}\right)$. The homotopy groups of the space $\mathbf{L N}_{n}\left(\phi_{V}\right)$ are the groups $L N_{n+*}\left(\phi_{V}\right)$, which are special cases of the $L S$ groups which are obstruction groups in codimension 1 surgery theory. The groups $L N_{n+*}\left(\phi_{V}\right)$ are well known to be isomorphic to the $L$-groups of the trivial group from 'ordinary' surgery theory. Thus we also obtain a calculation of the homotopy groups of the spaces $F^{(1)}(V, y)$.

Relating the comparison between the block structure spaces of real projective spaces of different dimensions with codimension 1 surgery theory was not our original idea. At the level of structure sets it was used by Browder-Livesay, López de Medrano and Wall in order to calculate the structure sets of the real projective spaces (see [BL, [LdM], Wa, chapter 14C]). The statements at the level of block structure spaces which we review here follow from general theory of $\mathrm{Wa}$, chapter $17 \mathrm{~A}]$ and from the treatment of codimension $q$ surgery theory in [Ra1, chapter 7].

In this section we use the definition of the block structure space $\widetilde{\mathcal{S}}^{s}(X)$ from Construction 1.6 and we work on the level of $\Delta$-sets. This is legal by Remarks 1.1 . 3.12. We also adopt the convention that an embedding of manifolds will always mean locally flat embedding. This condition is fulfilled in all cases that we treat here. 
4.1. Codimension $q$ surgery theory. We include a brief review of the codimension $q$ surgery theory in which we fix the terminology, the notation, and we also introduce some constructions that will be used in section 5. The reference for the general theory is Wa, chapter 11]. The treatment there is slightly more general; see remarks by the editor at the beginning of the chapter. Another useful reference is [Ra1, chapter 7]. We do assume however a basic knowledge of 'ordinary' surgery theory, in particular, the algebraic definition of the $L$-groups and the construction of the surgery obstruction for a degree one normal map (see Wa, chapters 5,6$]$ ).

First we need the following construction.

Construction 4.1. Let $M \hookrightarrow X$ be an embedding of manifolds. It induces the following decomposition of the ambient manifold $X$. The submanifold $M$ has a normal block bundle $\nu$, with total space $E(\nu)$ and with the total space of the associated sphere bundle denoted by $S(\nu)$. The closure of the complement of $E(\nu)$ in $X$ is denoted by $C$. Thus we have

$$
X=E(\nu) \cup_{S(\nu)} C .
$$

Definition 4.2. Let $M \hookrightarrow X$ be an embedding of manifolds with the normal block bundle $\nu$. A simple homotopy equivalence $f: Y \rightarrow X$ from a manifold $Y$ of the same dimension as $X$ is said to be split along $M$ w.r.t $\nu$ if $f$ is transverse to $M$ w.r.t. $\nu$, and the following restrictions are simple homotopy equivalences:

- $\left.f\right|_{M^{\prime}}: M^{\prime}=f^{-1}(M) \rightarrow M$,

- $\left.f\right|_{C^{\prime}}: C^{\prime}=f^{-1}(C) \rightarrow C$.

Definition 4.3. Let $M \hookrightarrow X$ be an embedding of manifolds as above. We will refer to a simple homotopy equivalence $f: Y \rightarrow X$ as a splitting problem (along $M$ ).

Given a splitting problem $f$ along $M$ the task is to change $f$ by a homotopy to a simple homotopy equivalence which is split along $M$. If $q=(\operatorname{dim}(X)-\operatorname{dim}(M)) \geq 3$ the obstruction theory reduces to the ordinary surgery theory with the $L$-groups as obstruction groups ([Wa, Theorem 11.3]). In cases $q=1,2$ the obstruction theory is different. First, we can assume that $\nu$ is a vector bundle. Then given a splitting problem $f$ along $M$ there is an obstruction $\theta(f)$ in a group $L S_{n}(\Phi)$. The obstruction vanishes if and only if the problem can be solved. Here $n=\operatorname{dim}(M)$, and $\Phi$ is the following diagram of fundamental groupoids:

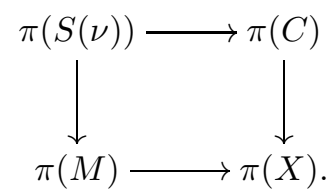

The groups $L S_{n}(\Phi)$ depend only on $\Phi$, dimension $n$, codimension $q$ and the orientation characters of $M$ and $X$. See [Wa, chapter 11] for the details of the construction of the $L S$-groups.

In the special case when $\pi(M) \rightarrow \pi(X)$ is an isomorphism the other horizontal morphism in $\Phi$ is also an isomorphism, and thus the diagram depends only on the vertical morphism $\phi: \pi(C) \rightarrow \pi(X)$. In this case the group $L S_{n}(\Phi)$ is denoted $L N_{n}(\phi)$. This will be the most important case for us.

Suppose now we are given an embedding of manifolds $M^{n} \hookrightarrow X^{n+q}$ which induces the diagram of fundamental groupoids $\Phi$. Following the general philosophy of $[\mathrm{Wa}$, chapter $17 \mathrm{~A}]$ to 'spacify' all obstruction groups in surgery theory we can 
define a space $\mathbf{L} \mathbf{S}_{n}(\Phi)$ such that $\pi_{k}\left(\mathbf{L} \mathbf{S}_{n}(\Phi)\right) \cong L S_{n+k}(\Phi)$ (see also [Ha]). In an outline, a $k$-simplex of the space $\mathbf{L S}_{n}(\Phi)$ is a splitting problem $Z \rightarrow Y$ of manifold $(k+2)$-ads along an embedding $N \hookrightarrow Y$ of manifold $(k+2)$-ads, which is solved on one part of the boundary and comes with a reference map $Y \rightarrow X$ which respects the decompositions of $Y$ and $X$ given by $N \hookrightarrow Y$ and $M \hookrightarrow X$ as described in Construction 4.1 .

We will not give a precise definition of this space in general; instead we look at the special case of an embedding $M^{n} \hookrightarrow E(\xi)$ of a manifold as the zero section of the total space of some disk bundle $\xi$. Let $\phi: \pi(S(\xi)) \rightarrow \pi(E(\xi))$ be the induced homomorphism of fundamental groupoids. The embedding corresponds to the $L N$ situation, so we will talk about the space $\mathbf{L N}_{n+1}(\phi)$.

Note that we are provided with a map $\xi^{!}: \widetilde{\mathcal{S}}^{s}(M) \rightarrow \widetilde{\mathcal{S}}^{s}(E(\xi), S(\xi))$. On a $k$ simplex $f: M^{\prime} \rightarrow M \times \Delta^{k}$ it is given by pulling back the disk bundle $E(\xi) \rightarrow M$ along $f$. Let $y$ be a 0 -simplex in $\widetilde{\mathcal{S}}^{s}(E(\xi), S(\xi))$, that means $y$ is a simple homotopy equivalence $f_{y}:(E, S) \rightarrow(E(\xi), S(\xi))$ which can also be seen as a splitting problem along $M$. The splitting obstruction $\theta\left(f_{y}\right)$ lives in the group $L N_{n}(\phi)$. Denote

$$
\widetilde{\mathcal{S}}^{s}(\xi, y)=\operatorname{hofiber}_{y}\left(\xi^{!}: \widetilde{\mathcal{S}}^{s}(M) \rightarrow \widetilde{\mathcal{S}}^{s}(E(\xi), S(\xi))\right) .
$$

By definition (see [Ra2, chapter 11]) a $k$-simplex of $\widetilde{\mathcal{S}}^{s}(\xi, y)$ is a simple homotopy equivalence $f:(E, S) \times \Delta^{k+1} \rightarrow(E(\xi), S(\xi)) \times \Delta^{k+1}$ which respects faces, such that $f_{y}=\partial_{\alpha} f:(E, S) \times \partial_{\alpha} \Delta^{k+1} \rightarrow(E(\xi), S(\xi)) \times \partial_{\alpha} \Delta^{k+1}$ for $\alpha: \mathbf{0} \rightarrow \mathbf{k}+\mathbf{1}$, such that $\alpha(0)=k+1$, and $\partial_{k+1} f:(E, S) \times \partial_{k+1} \Delta^{k+1} \rightarrow(E(\xi), S(\xi)) \times \partial_{k+1} \Delta^{k+1}$ is split along $M$, where $k+1: \mathbf{k} \rightarrow \mathbf{k}+\mathbf{1}$ is obtained by omitting $k+1$ in the target.

It is not hard to see that

- if $\theta\left(f_{y}\right) \neq 0$, then $\widetilde{\mathcal{S}}^{s}(\xi, y)$ is empty,

- if $\theta\left(f_{y}\right)=0$, then $\widetilde{\mathcal{S}}^{s}(\xi, y) \simeq \mathbf{L N}_{n+1}(\phi)$.

For the second statement, note that a $k$-simplex of the space $\widetilde{\mathcal{S}}^{s}(\xi, y)$ is a splitting problem of manifold $(k+3)$-ads which is solved on the part $\partial_{\alpha} \cup \partial_{k+1}$ of the boundary. As such it can be considered as a $k$-simplex of the space $\mathbf{L N}_{n+1}(\phi)$, which gives an inclusion map which is the indicated homotopy equivalence; it induces isomorphisms on homotopy groups. See for example [Ra1, chapter 7.2] for the reference.

4.2. The homotopy type of $F^{(1)}(V, y)$. Now consider the embedding $\mathbb{R} P(V) \hookrightarrow$ $\mathbb{R} P(V \oplus \mathbb{R})$. We have the decomposition

$$
\mathbb{R} P(V \oplus \mathbb{R})=E(\nu) \cup_{S(\nu)} C,
$$

where $\nu$ is the normal disk bundle of $\mathbb{R} P(V)$ and $C \cong D^{n}, n=\operatorname{dim}(V)$.

Now consider the embedding $\mathbb{R} P(V) \hookrightarrow E(\nu)$, the induced homomorphism $\phi_{V}: \pi_{1}(S(\nu)) \rightarrow \pi_{1}(E(\nu))$ and a homotopy equivalence $y=f_{y}:(E, S) \rightarrow(E(\nu), S(\nu))$ with the splitting obstruction $\theta\left(f_{y}\right) \in L N_{n-1}\left(\phi_{V}\right)$. Consider

$$
\widetilde{\mathcal{S}}^{s}(\nu, y)=\operatorname{hofiber}_{y}\left(\nu^{!}: \widetilde{\mathcal{S}}^{s}(\mathbb{R} P(V)) \rightarrow \widetilde{\mathcal{S}}^{s}(E(\nu), S(\nu))\right) .
$$

By the above discussion we have that

- if $\theta\left(f_{y}\right) \neq 0$, then $\widetilde{\mathcal{S}}^{s}(\nu, y)$ is empty,

- if $\theta\left(f_{y}\right)=0$, then $\widetilde{\mathcal{S}}^{s}(\nu, y) \simeq \mathbf{L N}_{n}\left(\phi_{V}\right)$.

Now let $y=f_{y}: M \rightarrow \mathbb{R} P(V \oplus \mathbb{R})$ be a 0 -simplex in $\widetilde{\mathcal{S}}^{s}(\mathbb{R} P(V \oplus \mathbb{R}))$. Consider the space $F^{(1)}(V, y)=\operatorname{hofiber}_{y}\left(\widetilde{\mathcal{S}}^{s}(\mathbb{R} P(V)) \rightarrow \widetilde{\mathcal{S}}^{s}(\mathbb{R} P(V \oplus \mathbb{R}))\right)$. A $k$-simplex of this space can be viewed as a splitting problem over $\mathbb{R} P(V \oplus \mathbb{R}) \times \Delta^{k+1}$ along 
$\mathbb{R} P(V) \times \Delta^{k+1}$ satisfying certain conditions on the boundary. The homotopy type of $F^{(1)}(V, y)$ is identified in the following proposition.

Proposition 4.4. Let $\operatorname{dim}(V)=n \geq 6$ and consider the homotopy equivalence $y=f_{y}: M \rightarrow \mathbb{R} P(V \oplus \mathbb{R})$ as a splitting problem along $\mathbb{R} P(V)$ with the splitting obstruction $\theta\left(f_{y}\right) \in L N_{n-1}\left(\phi_{V}\right)$. Then the following hold:

- if $\theta\left(f_{y}\right) \neq 0$, then $F^{(1)}(V, y)$ is empty,

- if $\theta\left(f_{y}\right)=0$, then $F^{(1)}(V, y) \simeq \mathbf{L N}_{n}\left(\phi_{V}\right)$.

Proof. First note that there is a map $\widetilde{\mathcal{S}}^{s}(E(\nu), S(\nu)) \rightarrow \widetilde{\mathcal{S}}^{s}(\mathbb{R} P(V \oplus \mathbb{R}))$ given by 'coning off'. This map is a homotopy equivalence by the following argument.

By transversality there is the following homotopy cartesian square:

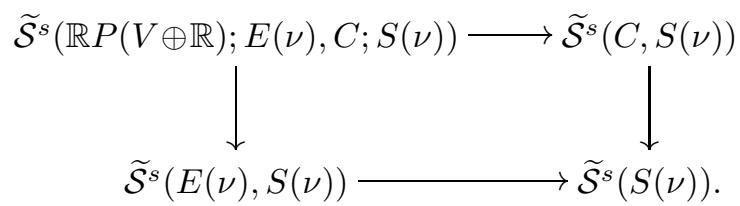

By the result of [Wa, Theorem 12.1], if $\operatorname{dim}(V) \geq 6$, then the forgetful map $\widetilde{\mathcal{S}}^{s}(\mathbb{R} P(V \oplus \mathbb{R}) ; E(\nu), C ; S(\nu)) \rightarrow \widetilde{\mathcal{S}}^{s}(\mathbb{R} P(V \oplus \mathbb{R}))$ is a homotopy equivalence. It is also well known that the forgetful map $\widetilde{\mathcal{S}}^{s}(C, S(\nu)) \rightarrow \widetilde{\mathcal{S}}^{s}(S(\nu))$ is a homotopy equivalence. Therefore the left-hand vertical map is also a homotopy equivalence. The 'coning off' map is its homotopy inverse.

Abusing notation we also denote by $y$ the image of $y \in \widetilde{\mathcal{S}}^{s}(\mathbb{R} P(V \oplus \mathbb{R}))$ under some homotopy inverse of the coning off map. We have the following diagram which commutes up to homotopy:

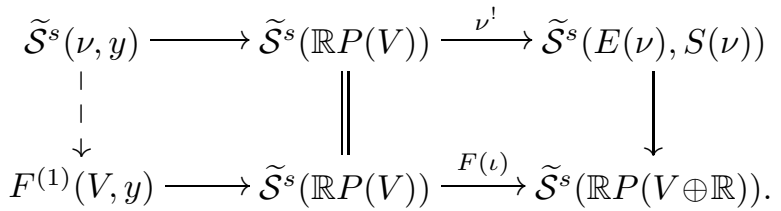

The left-hand vertical map is induced by the right-hand vertical map which is a homotopy equivalence, and so the left-hand map is also a homotopy equivalence.

By the criterion preceding the theorem we get the desired statement.

Proposition 4.5. If $\operatorname{dim}(V)=n \geq 6$ we have

$$
\pi_{k}\left(\mathbf{L N}_{n}\left(\phi_{V}\right)\right) \cong L N_{n+k}\left(\phi_{V}\right) \cong L_{k-n}(1) .
$$

Proof. We have the homomorphism $\phi_{V}: 1 \rightarrow \mathbb{Z}_{2}$, where the subscript $V$ stands for the orientation character. This equals $(-1)^{n}$. By Wa, Corollary 12.9.1] we have that $L N_{*}\left(1 \rightarrow \mathbb{Z}_{2}\right) \cong L_{*+\varepsilon}(1)$, where $\varepsilon=0$ if the orientation character is -1 and $\varepsilon=2$ if the orientation character is +1 . This together with the 4-periodicity of the $L$-groups yields the desired statement.

4.3. An isomorphism from $\pi_{k}\left(\mathbf{L N}_{n}\left(\phi_{V}\right)\right)$ to $L_{k-n}(1)$. Following Wa, chapter $12 \mathrm{C}$ ] we now give a concrete and explicit description of the isomorphism

$$
\theta: \pi_{k}\left(\mathbf{L N}_{n}\left(\phi_{V}\right)\right) \rightarrow L_{k-n}(1) .
$$


By definition a $k$-simplex in $\mathbf{L N}_{n}\left(\phi_{V}\right)$ is a splitting problem $f:(E, S) \times \Delta^{k+1} \rightarrow$ $(E(\nu), S(\nu)) \times \Delta^{k+1}$ along $\mathbb{R} P(V) \times \Delta^{k+1}$ satisfying certain conditions on the boundary. The isomorphism $\theta$ sends the homotopy class of $f$ to the splitting obstruction rel $\partial \Delta^{k+1}$ of $f$ which lives in the group $L_{k-n}(1)$. Simultaneously we give a description of the map

$$
\theta: \pi_{0}\left(\widetilde{\mathcal{S}}^{s}(E(\nu), S(\nu))\right) \rightarrow L_{-1-n}(1)
$$

A 0 -simplex of $\widetilde{\mathcal{S}}^{s}(E(\nu), S(\nu))$ is a splitting problem $f:(E, S) \rightarrow(E(\nu), S(\nu))$ along $\mathbb{R} P(V)$, and the map $\theta$ sends $f$ to its splitting obstruction.

So we consider a splitting problem $f:(E, S) \times \Delta^{k+1} \rightarrow(E(\nu), S(\nu)) \times \Delta^{k+1}$, where $k \geq-1$, and we find a splitting obstruction $\theta(f)$ in $L_{k-n}(1)$. If $(k-n)$ is odd, then $L_{k-n}(1)$ is trivial, so it is enough to consider the case when $(k-n)$ is even and hence also $(n+k)$ is even.

The even-dimensional $L$-groups are defined algebraically in WW, chapter 5]. For a group $\pi$ with a homomorphism $w: \pi \rightarrow \mathbb{Z}_{2}$ and for $2 m=(k-n)$ an element of the group $L_{k-n}(\pi)$ is represented by a simple $(-1)^{m}$-hermitian form $(G, \lambda, \mu)$, where $G$ is a stably free $\mathbb{Z}[\pi]$-module and the maps

$$
\lambda: G \times G \rightarrow \mathbb{Z}[\pi], \quad \mu: G \rightarrow \mathbb{Z}[\pi] /\left\{x-(-1)^{l} \bar{x}\right\},
$$

where $x \mapsto \bar{x}$ is an involution on $\mathbb{Z}[\pi]$ induced by the homomorphism $w$, satisfy certain conditions; see $\mathrm{Wa}$, Theorem 5.2]. A submodule $H$ of $G$ is called a lagrangian of the form $(G, \lambda, \mu)$ if the map $\lambda$ induces an isomorphism $G / H \rightarrow \operatorname{Hom}_{\mathbb{Z}[\pi]}(H, \mathbb{Z}[\pi])$, $\lambda(H \times H)=0$ and $\mu(H)=0$. The form $(G, \lambda, \mu)$ admits a lagrangian if and only if it represents a zero element in the $L$-group in which case it is called a hyperbolic form.

In Wa, chapter 5] the surgery obstruction for converting an even-dimensional degree one normal map into a simple homotopy equivalence is described as an element of such an $L$-group. Following [Wa, chapter $12 \mathrm{C}$ ] we now describe how to associate a simple $(-1)^{m}$-hermitian form which represents an element in $L_{k-n}(1)$ to the splitting problem $f$ as above.

Construction 4.6. So we start with $f:(E, S) \times \Delta^{k+1} \rightarrow(E(\nu), S(\nu)) \times \Delta^{k+1}$, a homotopy equivalence satisfying appropriate conditions on the boundary. Denote by $N=f^{-1}\left(\mathbb{R} P(V) \times \Delta^{k+1}\right)$ and by $i$ the embedding $N \hookrightarrow(E, S) \times \Delta^{k+1}$, and note that $i$ is a degree one normal map. The obstruction $\theta(f)$ coincides with the obstruction to make the embedding $i$ into a homotopy equivalence by an ambient surgery on $N$ inside $(E, S) \times \Delta^{k+1}$ (see $[\mathrm{Wa}$, chapter 12C] or [Ra1, chapter 7.6] for the definition of the ambient surgery on a submanifold).

This is defined as follows. Let $2 l=(n+k)$. First make $i$ into an $l$-connected map by an ambient surgery. The ordinary surgery obstruction associated to $i$ is a simple $(-1)^{l}$-hermitian form $(H, \lambda, \mu)$ over $\mathbb{Z}\left[\mathbb{Z}_{2}\right]$, where $H=\pi_{l+1}(i)=\pi_{l+1}\left(E \times \Delta^{k+1}, N\right)$. As usual the elements of $H$ can be represented by $l$-dimensional spheres immersed in $N$, the form $\lambda$ is given by the $\mathbb{Z}\left[\mathbb{Z}_{2}\right]$-intersection numbers of these spheres and $\mu$ is given by the $\mathbb{Z}\left[\mathbb{Z}_{2}\right]$-self-intersection numbers. Let us now instead look at the double covers and try to change $\widetilde{i}: \widetilde{N} \hookrightarrow \widetilde{E} \times \Delta^{k+1}$ by an equivariant ambient surgery. Here $\widetilde{E}$ denotes the non-trivial double cover of $E$. The ordinary non-equivariant surgery obstruction associated to $\widetilde{i}$ is a simple $(-1)^{l}$-hermitian form $\left(H, \lambda^{\prime}, \mu^{\prime}\right)$ over $\mathbb{Z}$, with the same $H$, only considered now as a $\mathbb{Z}$-module. The maps $\lambda^{\prime}, \mu^{\prime}$ are now given by $\mathbb{Z}$-intersection, resp. $\mathbb{Z}$-self-intersection numbers of the representing immersed spheres. The form $\left(H, \lambda^{\prime}, \mu^{\prime}\right)$ is hyperbolic. More to the point, $\widetilde{N}$ is a separating 
submanifold, and we have a decomposition $\widetilde{E} \times \Delta^{k+1}=A^{+} \cup_{\widetilde{N}} A^{-}$. The $\mathbb{Z}$-module $H=\pi_{l+1}(\widetilde{i})=\pi_{l+1}\left(\widetilde{E} \times \Delta^{k+1}, \widetilde{N}\right)$ splits as

$$
H^{+} \oplus H^{-}=\pi_{l+1}\left(A^{+}, \widetilde{N}\right) \oplus \pi_{l+1}\left(A^{-}, \widetilde{N}\right) .
$$

The involution $T$ switches $H^{+}$and $H^{-}$, which are both lagrangians of $\left(H, \lambda^{\prime}, \mu^{\prime}\right)$.

The equivariant ambient surgery obstruction for $\widetilde{i}$ is now defined as follows. Denote by $\lambda_{0}(x, y)=\lambda^{\prime}(x, t y), \mu_{0}(x)=t \mu(x)$, where $t$ is the non-trivial element of $\mathbb{Z}_{2}$. Then $\left(H^{+}, \lambda_{0}, \mu_{0}\right)$ is a simple $(-1)^{m}$-hermitian form over $\mathbb{Z}$ where $2 m=(k-n)$. It represents the desired obstruction $\theta(f)$ in $L_{k-n}(1)$.

Furthermore we may assume that $H^{+} \cong \pi_{l+1}\left(A^{+}, \widetilde{N}\right)$ is a free $\mathbb{Z}$-module on generators $\left\{e_{i}\right\}_{i \in \mathbf{r}}$ whose boundaries are represented by the spheres $S_{i}^{l}$ disjointly embedded in $\widetilde{N}$ (because $H^{+}$is a lagrangian over $\mathbb{Z}$ ). Let $\widetilde{N} \times I$ be the normal disk bundle of $\widetilde{N}$ in $\widetilde{E} \times \Delta^{k+1}$. Then the generators $e_{i}$ can be represented by the disks $D_{i}^{l+1}$ disjointly embedded in $\widetilde{E} \times \Delta^{k+1}$ so that the disk $D_{i}^{l+1}$ is attached to the normal bundle $\widetilde{N} \times I$ at the sphere $S_{i}^{l} \times\{1\}$ (see [BL]). Similarly $H^{-}$is generated by the elements $\left\{t e_{i}\right\}_{i \in \mathbf{r}}$ whose boundaries are represented by the spheres $T S_{i}^{l}$, and the elements $t e_{i}$ are themselves represented by the disks $T D_{i}^{l+1}$ attached to $\widetilde{N} \times I$ at $T S_{i}^{l} \times\{-1\}$.

Remark 4.7. We did not prove that $\theta$ is an isomorphism if $k \geq 0$; we have only given a description of the map $\theta$. However, from the fact that $\theta$ is an isomorphism if $k \geq 0$ follows an important realization theorem which says that if $k \geq 0$, then any element of $L_{k-n}(1)$ can be represented by a splitting problem over $(E(\nu), S(\nu)) \times \Delta^{k+1}$ along $\mathbb{R} P(V) \times \Delta^{k+1}$. We refer to [Wa, chapter $\left.12 \mathrm{C}\right]$ for the details.

Remark 4.8. In this section we have used a model of $F^{(1)}(V, y)$ such that a $k$-simplex was a splitting problem over $\mathbb{R} P(V \oplus \mathbb{R}) \times \Delta^{k+1}$ along $\mathbb{R} P(V) \times \Delta^{k+1}$ satisfying certain conditions on the boundary. In the next section it will be more convenient to use another model where a $k$-simplex is an equivariant splitting problem over $S(V \oplus \mathbb{R}) \times \Delta^{k+1}$ along $S(V) \times \Delta^{k+1}$ (it is clear what involutions are meant) satisfying corresponding conditions on the boundary.

\section{The FunCtor $F$ IS POLYNOMial of DEGREe $\leq 1$}

In this section we prove the two main theorems of this paper, Theorem 5.1 and Theorem [5.11] We start with:

Theorem 5.1. Let $F: \mathcal{J} \rightarrow$ Spaces $_{*}$ be the functor defined by $V \mapsto \widetilde{\mathcal{S}}^{s}(\mathbb{R} P(V))$. If $\operatorname{dim}(V) \geq 6$, then the canonical maps

$$
F(V) \rightarrow \operatorname{holim}_{0 \neq U \subset \mathbb{R}^{2}} F(V \oplus U)
$$

are homotopy equivalences.

An immediate corollary of Theorem 5.1 is the following.

Corollary 5.2. If $\operatorname{dim}(V) \geq 6$, then the canonical maps

$$
F(V) \rightarrow T_{1} F(V)
$$

are homotopy equivalences. 
Proof of Corollary 5.2. The definition of the functor $T_{1} E$ for a general continuous functor $E: \mathcal{J} \rightarrow$ Spaces $_{*}$ is given in [We, Theorem 6.3]. It says that $T_{1} E$ is the homotopy colimit of the direct system

$$
E \stackrel{\rho}{\rightarrow} \tau_{1} E \stackrel{\tau_{1}(\rho)}{\longrightarrow}\left(\tau_{1}\right)^{2} E \stackrel{\left(\tau_{1}\right)^{2}(\rho)}{\longrightarrow} \cdots,
$$

where

$$
\tau_{1} E(V)=\operatorname{holim}_{0 \neq U \subset \mathbb{R}^{2}} E(V \oplus U),
$$

and the natural transformation $\rho: E \rightarrow \tau_{1} E$ is given by the canonical maps. Theorem 5.1 says that in the case of the functor $F$ the maps $\rho: F(V) \rightarrow \tau_{1} F(V)$ are homotopy equivalences if $\operatorname{dim}(V) \geq 6$. The claim of Corollary 5.2 follows.

Overview of the proof of Theorem 5.1. The details of the proof of Theorem 5.1 are rather complicated. Therefore we first give an overview of the proof.

The pattern of the proof is to verify the condition of Theorem 3.6 for the map (5.1) to be a homotopy equivalence, if $\operatorname{dim}(V) \geq 6$. This condition says that we have to verify that a certain map $\tilde{\sigma}_{1}: F^{(1)}(V, y) \rightarrow X_{y}$ is a homotopy equivalence for all choices of $y \in F(V \oplus \mathbb{R})$.

The space $X_{y}$ and the map $\tilde{\sigma}_{1}$ were described for a general continuous functor $E: \mathcal{J} \rightarrow$ Spaces $_{*}$ in Construction 3.5. We work with the $\Delta$-set models, so in Construction 5.3 we provide a $\Delta$-set model of the space $X_{y}$ and we also provide a $\Delta$-set description of the map $\tilde{\sigma}_{1}$ which up to homotopy commutes with the geometric realization.

The homotopy type of the space $F^{(1)}(V, y)$ has been described in Proposition 4.4. It says that $F^{(1)}(V, y)$ is empty if and only if the splitting obstruction $\theta\left(f_{y}\right)$ of a certain equivariant splitting problem $f_{y}$ over $S(V \oplus \mathbb{R})$ along $S(V)$ is non-zero (see also Remark 4.8). If $\theta\left(f_{y}\right)=0$, then the space $F^{(1)}(V, y)$ is homotopy equivalent to a certain space $\mathbf{L N}_{n}\left(\phi_{V}\right)$ whose homotopy groups are calculated in Proposition 4.5 .

We give a corresponding description of the homotopy type of the space $X_{y}$ in Proposition 5.4. It says that $X_{y}$ is empty if and only if the splitting obstruction $\theta\left(f_{y}^{\prime}\right)$ of a certain equivariant splitting problem $f_{y}^{\prime}$ over $S\left(V \oplus \mathbb{R}^{2}\right) \times I$ and along $S(V \oplus \mathbb{R}) \times I$ is non-zero. If $\theta\left(f_{y}^{\prime}\right)=0$, then the space $X_{y}$ is homotopy equivalent to the space $\Omega \mathbf{L} \mathbf{N}_{n+1}\left(\phi_{V \otimes \mathbb{R}}\right)$ whose homotopy groups are also calculated by Proposition 4.5 .

As we have indicated in Remark 3.8 the proof then splits into two parts. To prove condition (1) we have to show that the space $F^{(1)}(V, y)$ is empty if and only if the space $X_{y}$ is empty. This is the content of Proposition 5.5. It is proved using the criteria mentioned above. Note that the equivariant splitting obstructions $\theta\left(f_{y}\right)$ and $\theta\left(f_{y}^{\prime}\right)$ live in the same group $L_{-1-n}(1)$. Therefore it is enough to check that $\theta\left(f_{y}\right)=\theta\left(f_{y}^{\prime}\right)$ in $L_{-1-n}(1)$. The proof of this statement is the crucial part of the whole proof. Unfortunately it is rather technical. It is given in a sequence of lemmas: Lemma 5.7, 5.8, 5.9.

To prove condition (2) we have to show that if $F^{(1)}(V, y)$ is not empty, then the map $\tilde{\sigma}_{1}: F^{(1)}(V, y) \rightarrow X_{y}$ is a homotopy equivalence. This is done by showing that it induces isomorphisms on homotopy groups. Using Propositions 4.4, 5.4 and 4.5 we see that the spaces $F^{(1)}(V, y)$ and $X_{y}$ have isomorphic homotopy groups. Thus we have to show that the map $\tilde{\sigma}_{1}$ induces isomorphisms between abstractly isomorphic groups. The idea here is the following. An element of $\pi_{k}\left(F^{(1)}(V, y)\right) \cong$ 
$L_{k-n}(1)$ can be represented by a splitting obstruction $\theta(f)$ of some equivariant splitting problem $f$ over $S(V \oplus \mathbb{R}) \times \Delta^{k+1}$ along $S(V) \times \Delta^{k+1}$. The image $\tilde{\sigma}_{1}(f)$ is a splitting problem over $S\left(V \oplus \mathbb{R}^{2}\right) \times \Delta^{k+1} \times I$ along $S(V \oplus \mathbb{R}) \times \Delta^{k+1} \times I$ with the splitting obstruction $\theta\left(\tilde{\sigma}_{1}(f)\right)$ which represents the homotopy class of $\tilde{\sigma}_{1}(f)$ in $\pi_{k}\left(X_{y}\right) \cong L_{k-n}(1)$. Condition (2) is proved by showing that $\theta(f)=\theta\left(\tilde{\sigma}_{1}(f)\right)$ in $L_{k-n}(1)$. This statement is the content of Proposition 5.10 Its proof turns out to be just a parameterized version of the proof of the statement of condition (1).

Construction 5.3. We need a $\Delta$-set model of the space $X_{y}$ and a description of the map $\tilde{\sigma}_{1}$ on the level of $\Delta$-sets. The descriptions given here and the descriptions from Construction 3.5 commute with the geometric realization up to homotopy.

Recall the $\Delta$-set model for $F^{(1)}(V, y)$. Here $y=f_{y}: S(V \oplus \mathbb{R}) \rightarrow S(V \oplus \mathbb{R})$ is an equivariant splitting problem along $S(V)$. A $k$-simplex of $F^{(1)}(V, y)$ is an equivariant homotopy equivalence $f: S(V \oplus \mathbb{R}) \times \Delta^{k+1} \rightarrow S(V \oplus \mathbb{R}) \times \Delta^{k+1}$ w.r.t. the antipodal involution on the target and w.r.t. some free involution on the source, it respects faces, it is transverse to $S(V) \times \Delta^{k+1}$, for $\alpha: \mathbf{0} \rightarrow \mathbf{k}+\mathbf{1}, \alpha(0)=k+1$, we have $f_{y}=\partial_{\alpha} f: S(V \oplus \mathbb{R}) \times \partial_{\alpha} \Delta^{k+1} \rightarrow S(V \oplus \mathbb{R}) \times \partial_{\alpha} \Delta^{k+1}$ and $\partial_{k+1} f$ is equivariantly split along $S(V) \times \Delta^{k}$.

Now we give a $\Delta$-set model for $X_{y}$. A $k$-simplex of $X_{y}$ is an equivariant homotopy equivalence $f^{\prime}: S\left(V \oplus \mathbb{R}^{2}\right) \times \Delta^{k+1} \times I \rightarrow S\left(V \oplus \mathbb{R}^{2}\right) \times \Delta^{k+1} \times I$ w.r.t. the antipodal involution on the target and w.r.t. to some free involution on the source which respects faces and satisfies the following conditions on the boundary.

First define

$$
\begin{gathered}
\partial_{0}\left(\Delta^{k+1} \times I\right)=\left(\partial_{k+1} \Delta^{k+1} \times I\right) \cup\left(\Delta^{k+1} \times \partial I\right), \\
\partial_{1}\left(\Delta^{k+1} \times I\right)=\partial_{\alpha} \Delta^{k+1} \times I,
\end{gathered}
$$

where $\alpha: \mathbf{0} \rightarrow \mathbf{k}+\mathbf{1}$ is again given by $\alpha(0)=k+1$.

The conditions on $f^{\prime}$ are the following. First $f^{\prime}$ restricted to $S\left(V \oplus \mathbb{R}^{2}\right) \times$ $\partial_{0}\left(\Delta^{k+1} \times I\right)$ is split along $S(V \oplus \mathbb{R}) \times \partial_{0}\left(\Delta^{k+1} \times I\right)$. The restriction of $f^{\prime}$ to $S\left(V \oplus \mathbb{R}^{2}\right) \times \partial_{1}\left(\Delta^{k+1} \times I\right)$ is fixed, and it is given as the composition

$$
f_{y}^{\prime}=(S(\zeta))^{-1} \circ\left(\Sigma\left(f_{y}\right) \times \mathrm{id}\right) \circ S(\zeta) .
$$

Here $\Sigma$ means the unreduced suspension map, and the map $S(\zeta): S\left(V \oplus \mathbb{R}^{2}\right) \times I \rightarrow$ $S\left(V \oplus \mathbb{R}^{2}\right) \times I$ is a homeomorphism given by $(x, t) \mapsto\left(\zeta_{t}(x), t\right)$, where $\zeta_{t}$ is the unique rotation of $V \oplus \mathbb{R}^{2}$ which fixes $V$ and sends $(0,1,0)$ to $\left(0, t, \sqrt{1-t^{2}}\right)$.

The map $\tilde{\sigma}_{1}: F^{(1)}(V, y) \rightarrow X_{y}$ is given by the rule

$$
\tilde{\sigma}_{1}: f \mapsto(S(\zeta))^{-1} \circ(\Sigma(f) \times \mathrm{id}) \circ S(\zeta) .
$$

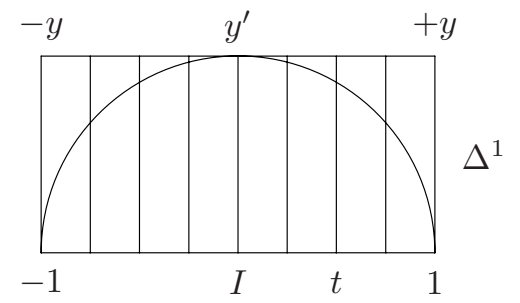

Figure 4. Schematic picture of a 0 -simplex in $X_{y}$ and of the map $\tilde{\sigma}_{1}$. 
A 0-simplex of $X_{y}$ is depicted in Figure 4. The top edge corresponds to the part $\partial_{1}\left(\Delta^{1} \times I\right)$ of the boundary. The closure of the complement of $\partial_{1}\left(\Delta^{1} \times I\right)$ in the boundary is $\partial_{0}\left(\Delta^{1} \times I\right)$. On each vertical line over $t \in I$ the restriction of the map $\tilde{\sigma}_{1}(f)$ is given by $\left(\zeta_{t}\right)^{-1} \circ \Sigma(f) \circ \zeta_{t}$. Compare this with Figure 3, The vertical lines here correspond after reparametrization to the rays from the origin in Figure 3 .

Note that the map $f_{y}^{\prime}$ can be seen as an equivariant splitting problem along $S(V \oplus \mathbb{R}) \times I$ with the splitting obstruction $\theta\left(f_{y}^{\prime}\right) \in L N_{n+1}\left(\phi_{V \oplus \mathbb{R}}\right) \cong L_{-1-n}(1)$. We need the following criterion.

Proposition 5.4. We have that

- if $\theta\left(f_{y}^{\prime}\right) \neq 0$, then the space $X_{y}$ is empty,

- if $\theta\left(f_{y}^{\prime}\right)=0$, then $X_{y} \simeq \Omega \mathbf{L} \mathbf{N}_{n+1}\left(\phi_{V \oplus \mathbb{R}}\right)$.

Proof. If $X_{y}$ is not empty, then there exists a 0 -simplex, say $f$, of $X_{y}$. Since $f$ restricted to $\left(S\left(V \oplus \mathbb{R}^{2}\right) \times \partial_{0}\left(\Delta^{k+1} \times I\right)\right)$ is by definition split along $S(V \oplus \mathbb{R}) \times$ $\partial_{0}\left(\Delta^{k+1} \times I\right)$, any such a 0 -simplex $f$ can be seen as a solution of the splitting problem $f_{y}^{\prime}$ and hence $\theta\left(f_{y}^{\prime}\right)=0$. As we have remarked in Construction 3.5 if the space $X_{y}$ is not empty it is homotopy equivalent to a certain space of paths in the space $F^{(1)}\left(V \oplus \mathbb{R}, y^{\prime}\right) \simeq \mathbf{L N}_{n+1}\left(\phi_{V \oplus \mathbb{R}}\right)$. It is not difficult to see that this path space is homotopy equivalent to $\Omega \mathbf{L} \mathbf{N}_{n+1}\left(\phi_{V \oplus \mathbb{R}}\right)$.

Now we are ready to proceed with a verification of the condition of Theorem 3.6 for the functor $F$ to be polynomial of degree $\leq 1$. We start with condition (1).

Proposition 5.5. $F^{(1)}(V, y)$ is empty if and only if $X_{y}$ is empty.

Propositions 4.45 .4 tell us when the spaces $F^{(1)}(V, y)$ and $X_{y}$ are empty. We see that both obstructions $\theta\left(f_{y}\right)$ and $\theta\left(f_{y}^{\prime}\right)$ live in the group $L_{-1-n}(1)$. In order to prove condition (1) it is enough to show that $\theta\left(f_{y}\right)=\theta\left(f_{y}^{\prime}\right)$ in $L_{-1-n}(1)$. This is done in the sequence of the following three lemmas.

Remark 5.6. Note that a precomposition with a homeomorphism does not change the splitting obstruction of a splitting problem. Therefore when investigating $\theta\left(f_{y}^{\prime}\right)$ it is enough to look at the splitting obstruction of the map $(S(\zeta))^{-1} \circ\left(\Sigma\left(f_{y}\right) \times\right.$ id $)$, and so from now on we denote

$$
f_{y}^{\prime}=(S(\zeta))^{-1} \circ\left(\Sigma\left(f_{y}\right) \times \mathrm{id}\right) .
$$

Lemma 5.7. The map $f_{y}^{\prime}: S\left(V \oplus \mathbb{R}^{2}\right) \times I \rightarrow S\left(V \oplus \mathbb{R}^{2}\right) \times I$ is transverse to the submanifold $S(V \oplus \mathbb{R}) \times I$.

Proof. One would expect that in order to find the splitting obstruction $\theta\left(f_{y}^{\prime}\right)$ it is necessary to first adjust the map $f_{y}^{\prime}$ to make it transverse to $S(V \oplus \mathbb{R}) \times I$. Amazingly, the map $f_{y}^{\prime}$ as constructed by the formula (5.4) is already transverse to $S(V \oplus \mathbb{R}) \times I$ ! This is what we prove in this lemma; more precisely we show an equivalent statement that the map $\left(\Sigma\left(f_{y}\right) \times\right.$ id $)$ is transverse to $S(\zeta) \circ j$ where $j$ denotes the embedding $S(V \oplus \mathbb{R}) \times I \hookrightarrow S\left(V \oplus \mathbb{R}^{2}\right) \times I$.

We use an easier notion of transversality which is good enough for our purposes. It goes as follows. Let $M \hookrightarrow X$ be a codimension 1 embedding. Suppose that there exist an open neighborhood $E$ of $M$ in $X$ and a submersion $g: E \rightarrow \mathbb{R}$ such that $g^{-1}(0)=M$. (This implies that $M$ has a trivial normal bundle.) We say that a map $f: Y \rightarrow X$ is transverse to $M$ if $g \circ f: f^{-1}(E) \rightarrow \mathbb{R}$ is a submersion in a neighborhood of $f^{-1}(M)$. Note that this condition can be verified locally. 
In our case, $X=S\left(V \oplus \mathbb{R}^{2}\right) \times I$ and $M$ is the image of $S(V \oplus \mathbb{R}) \times I$ under the embedding $S(\zeta) \circ j$. Let $g=h \circ(S(\zeta))^{-1}$ where $h: S\left(V \oplus \mathbb{R}^{2}\right) \times I \rightarrow \mathbb{R}$ is the 'height function' defined by the projection on the second coordinate of $\mathbb{R}^{2}$. (This should be suitably restricted to a neighborhood of $M$ in $X$.)

We use the decompositions (5.5), (5.6) into (overlapping) codimension 0 (noncompact) submanifolds

$$
S(V \oplus \mathbb{R})=E(\widetilde{\nu}) \cup C_{ \pm},
$$

where $E(\widetilde{\nu})$ is the total space of the open normal vector bundle $\widetilde{\nu}$ of $S(V)$ in $S(V \oplus \mathbb{R})$, and $C_{ \pm}=D_{ \pm}(V \oplus \mathbb{R}) \backslash S(V)$, where $D_{ \pm}(V \oplus \mathbb{R})=S(V \oplus \mathbb{R}) \cap\left(V \oplus \mathbb{R}_{ \pm}\right)$ with $\mathbb{R}_{ \pm}=\{u \in \mathbb{R} \mid \pm u \geq 0\}$, and

$$
S\left(V \oplus \mathbb{R}^{2}\right)=E(\widetilde{\mu}) \cup D_{ \pm}
$$

where $E(\widetilde{\mu})$ is the total space of the open normal vector bundle $\widetilde{\mu}$ of $S(V)$ in $S\left(V \oplus \mathbb{R}^{2}\right)$, and $D_{ \pm}=D_{ \pm}\left(V \oplus \mathbb{R}^{2}\right) \backslash S(V)$, where $D_{ \pm}\left(V \oplus \mathbb{R}^{2}\right)=S\left(V \oplus \mathbb{R}^{2}\right) \cap\left(V \oplus \mathbb{R} \oplus \mathbb{R}_{ \pm}\right)$.

Note that the embedding $S(\zeta) \circ j$ restricts to the embeddings

$$
E(\widetilde{\nu}) \times I \hookrightarrow E(\widetilde{\mu}) \times I, \quad C_{ \pm} \times I \hookrightarrow D_{ \pm} \times I .
$$

We give two different arguments for the transversality on these two kinds of codimension 0 submanifolds of $S\left(V \oplus \mathbb{R}^{2}\right) \times I$

We start with the embedding $E(\widetilde{\nu}) \times I \hookrightarrow E(\widetilde{\mu}) \times I$. This is an embedding of a codimension 1 subbundle where both bundles are over $S(V) \times I$. The map $g$ restricted to $E(\widetilde{\mu}) \times I$ can be described as $g:(x, t) \mapsto\left(x-\operatorname{pr}_{l}(x)\right)$, where $\operatorname{pr}_{l}$ is the orthogonal projection in the fiber of $E(\widetilde{\mu}) \times\{t\}$ onto the image of $E(\widetilde{\nu}) \times\{t\}$.

Now the map $f_{y}$ was transverse to $S(V)$, therefore the map $\Sigma\left(f_{y}\right)$ is also transverse to $S(V)$. Let $\widetilde{N}:=\left(f_{y}\right)^{-1}(S(V))$ and let $\widetilde{\nu}_{\widetilde{N}}$ be the open normal bundle of $\widetilde{N}$ in $S(V \oplus \mathbb{R})$ and denote by $\widetilde{\mu}_{\widetilde{N}}$ the open normal bundle of $\widetilde{N}$ in $S\left(V \oplus \mathbb{R}^{2}\right)$. The restriction $\Sigma\left(f_{y}\right) \mid: E\left(\widetilde{\mu}_{\widetilde{N}}\right) \rightarrow E(\widetilde{\mu})$ is a bundle map. Now it is not difficult to see that the restriction $\Sigma\left(f_{y}\right) \mid \times$ id: $E\left(\widetilde{\mu}_{\widetilde{N}}\right) \times I \rightarrow E(\widetilde{\mu}) \times I$ is transverse to $E(\widetilde{\nu}) \times I$ in the above sense.

Next we look at the embedding $C_{ \pm} \times I \hookrightarrow D_{ \pm} \times I$. Observe that the composition $C_{ \pm} \times I \hookrightarrow D_{ \pm} \times I \rightarrow D_{ \pm}$is a homeomorphism. Therefore we can consider the embedding $C_{ \pm} \times I \cong D_{ \pm} \hookrightarrow D_{ \pm} \times I$ as an embedding of a graph of some function $\omega: D_{ \pm} \rightarrow I$ into $D_{ \pm} \times I$. The restriction of $g$ can be expressed as $(x, t) \mapsto t-\omega(x)$. Again it is not difficult to see that the restriction $\Sigma\left(f_{y}\right) \mid \times \mathrm{id}:\left(D_{ \pm}\left(V \oplus \mathbb{R}^{2}\right) \backslash \widetilde{N}\right) \times I \rightarrow$ $D_{ \pm} \times I$ is transverse to $C_{ \pm} \times I$ in the above sense.

The details are left to the reader.

In the next lemma we identify the homotopy type of $\widetilde{N}^{\prime}:=\left(f_{y}^{\prime}\right)^{-1}(S(V \oplus \mathbb{R}) \times I)$.

Lemma 5.8. Let $\tau: \tilde{N}^{\prime} \rightarrow \mathbb{R}$ be the composition $\tilde{N}^{\prime} \hookrightarrow S\left(V \oplus \mathbb{R}^{2}\right) \times I \rightarrow I \hookrightarrow \mathbb{R}$. Then $\tau$ is transverse to $s \in \mathbb{R}$ for all $s \neq 0$. Hence $\widetilde{N}^{\prime} \simeq \tau^{-1}(0) \cong \Sigma \widetilde{N}$, where $\Sigma \widetilde{N}$ means the unreduced suspension of $\widetilde{N}$.

Proof. A key observation here is that the manifold (with boundary) $\tilde{N}^{\prime}$ has a decomposition

$$
\widetilde{N}^{\prime}=E\left(\widetilde{\nu}_{\widetilde{N}}\right) \times I \cup\left(D_{ \pm}\left(V \oplus \mathbb{R}^{2}\right) \backslash \widetilde{N}\right)
$$

into codimension 0 submanifolds which corresponds to the previous decomposition $S(V \oplus \mathbb{R}) \times I=(E(\widetilde{\nu}) \times I) \cup D_{ \pm}$. This can be seen as follows. 
We can think of $\widetilde{N}^{\prime}$ as a pullback of $S(\zeta) \circ j$ along $\Sigma\left(f_{y}\right) \times$ id:

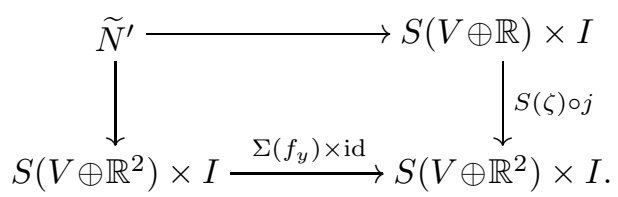

Look first at the following restriction of this diagram:

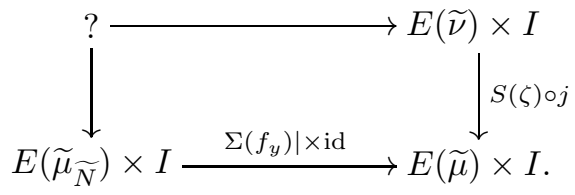

In this diagram the lower horizontal arrow is a bundle map over the restriction $f_{y} \mid \times$ id: $\widetilde{N} \times I \rightarrow S(V) \times I$. Here $E\left(\widetilde{\mu}_{\widetilde{N}}\right) \times I$ is a 2 -dimensional vector bundle over $\widetilde{N} \times I, E(\widetilde{\mu}) \times I$ is a 2-dimensional vector bundle over $S(V) \times I$ and $E(\widetilde{\nu}) \times I$ is its codimension 1 subbundle. It follows that the top row ? $\rightarrow E(\widetilde{\nu}) \times I$ is a bundle map, and it also follows from the construction that? can be identified with $E\left(\widetilde{\nu}_{\widetilde{N}}\right) \times I$.

Second, look at the restriction of the diagram to $C_{ \pm} \times I \cong D_{ \pm}$. Here, because the map $S(\zeta) \circ j$ is a map over $I$, we can look at the following pullback diagram:

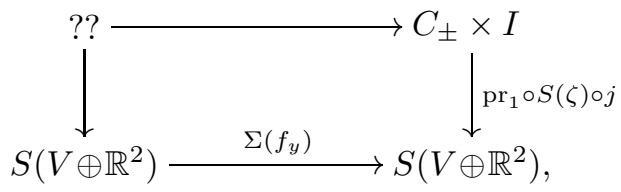

where $\mathrm{pr}_{1}$ is the projection onto the first factor. As noted before the composition $\left(\operatorname{pr}_{1} \circ S(\zeta) \circ j\right): C_{ \pm} \times I \rightarrow D_{ \pm}$is a homeomorphism, and thus we obtain that $? ? \cong \Sigma\left(f_{y}\right)^{-1}\left(D_{ \pm}\right)=\left(D_{ \pm}\left(V \oplus \mathbb{R}^{2}\right) \backslash \widetilde{N}\right)$. This yields the decomposition (5.7).

We describe the map $\tau$ on each submanifold in the decomposition. The restriction of $\tau$ to the submanifold $E\left(\widetilde{\nu}_{\widetilde{N}}\right) \times I$ is just the projection

$$
\tau \mid: E\left(\widetilde{\nu}_{\widetilde{N}}\right) \times I \rightarrow I \hookrightarrow \mathbb{R}
$$

which is clearly a submersion.

On the two submanifolds $D_{ \pm}\left(V \oplus \mathbb{R}^{2}\right) \backslash \widetilde{N}$ the restriction of the map $\tau$ can be described as follows. Observe first that $D_{ \pm}\left(V \oplus \mathbb{R}^{2}\right) \backslash \widetilde{N} \subset D_{ \pm}\left(V \oplus \mathbb{R}^{2}\right), D_{ \pm} \subset$ $D_{ \pm}\left(V \oplus \mathbb{R}^{2}\right)$ and that $D_{ \pm}\left(V \oplus \mathbb{R}^{2}\right)$ can be seen as a cone on $S(V \oplus \mathbb{R})$. The restriction of $\tau$ is the following composition:

$$
\tau \mid: D_{ \pm}\left(V \oplus \mathbb{R}^{2}\right) \backslash \widetilde{N} \stackrel{C\left(f_{y}\right)}{\longrightarrow} D_{ \pm} \cong C_{ \pm} \times I \rightarrow I \hookrightarrow \mathbb{R}
$$

where $C\left(f_{y}\right)$ denotes the canonical extension of $f_{y}$ to the cones suitably restricted. The homeomorphism $D_{ \pm} \cong C_{ \pm} \times I$ can be described as follows. The fiber $C_{ \pm} \times\{s\}$ is embedded in $D_{ \pm}$as an open 'meridian' through the point $\left(0, s, \pm \sqrt{1-s^{2}}\right)$. It is not difficult to see that the cone map $C\left(f_{y}\right)$ is transverse to all such meridians except the one corresponding to $s=0$ (details are left to the reader).

The identification $\tau^{-1}(0) \cong \Sigma \widetilde{N}$ follows from the construction of the map $f_{y}^{\prime}$ by formula (5.4). 
Lemma 5.9. Let $2 m=-1-n$ and let $\theta\left(f_{y}\right) \in L_{-1-n}(1)$ be represented by a simple $(-1)^{m}$-hermitian form $\left(H^{+}, \lambda_{0}, \mu_{0}\right)$. Then $\theta\left(f_{y}^{\prime}\right) \in L_{-1-n}(1)$ is represented by the same simple $(-1)^{m}$-hermitian form.

Proof. Recall that $f_{y}: S(V \oplus \mathbb{R}) \rightarrow S(V \oplus \mathbb{R})$ is a homotopy equivalence about which we can assume that it is transverse to $S(V)$ and also that it is obtained from a homotopy equivalence $E(\widetilde{\nu}) \rightarrow E(\widetilde{\nu})$ by 'coning off' (by Proposition 4.4). Here $E(\widetilde{\nu})$ denotes the total space of the normal disk bundle of $S(V)$ in $S(V \oplus \mathbb{R})$. The splitting obstruction $\theta\left(f_{y}\right)$ is represented by a simple $(-1)^{m}$-hermitian form $\left(H^{+}, \lambda_{0}, \mu_{0}\right)$ obtained as an equivariant ambient surgery obstruction associated to the embedding $\widetilde{i}: \widetilde{N}=\left(f_{y}\right)^{-1}(S(V)) \hookrightarrow E(\widetilde{\nu})$ (see Construction 4.6).

The homotopy equivalence $f_{y}^{\prime}: S\left(V \oplus \mathbb{R}^{2}\right) \times I \rightarrow S\left(V \oplus \mathbb{R}^{2}\right) \times I$ is constructed by formula (5.4). Let $E\left(\widetilde{\nu}^{\prime}\right)$ be the total space of the normal disk bundle of $S(V \oplus \mathbb{R})$ in $S\left(V \oplus \mathbb{R}^{2}\right)$ and note that $f_{y}^{\prime}$ is already in the form of a homotopy equivalence obtained from another homotopy equivalence $E\left(\widetilde{\nu}^{\prime}\right) \times I \rightarrow E\left(\widetilde{\nu}^{\prime}\right) \times I$ by 'coning off', so there is no need to adjust it. The splitting obstruction $\theta\left(f_{y}^{\prime}\right)$ should now be read off as the equivariant ambient surgery obstruction from the embedding $\widetilde{i^{\prime}}: \widetilde{N}^{\prime} \hookrightarrow E\left(\widetilde{\nu}^{\prime}\right) \times I$ by the recipe given in Construction 4.6.

In Lemma 5.8 we made a first step by identifying the homotopy type of $\widetilde{N}^{\prime}$. Moreover it follows from the construction of the map $f_{y}^{\prime}$ by formula (5.4) that the embedding $\widetilde{i^{\prime}}$ when restricted to $\tau^{-1}(0)$ is just the unreduced suspension map $\widetilde{\Sigma i}$. So we have a commutative diagram

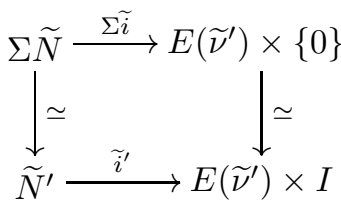

where all maps are embeddings.

Let $2 l=n-1$. From the diagram above it follows that $\widetilde{i}^{\prime}$ is $(l+1)$-connected, and also that $\pi_{l+2}\left(\widetilde{i^{\prime}}\right) \cong \pi_{l+1}(\widetilde{i}) \cong H$ and that the corresponding splitting of $H$ is the same $H^{+} \oplus H^{-}$as before. It only remains to find the embedded spheres representing the generators of $H$ and to show that their intersection numbers are preserved.

Let $\left\{e_{i}, t e_{i}\right\}$ be the set of generators of $H$ as a $\mathbb{Z}$-module, where generators are represented by embedded spheres $S_{i}, T S_{i} \hookrightarrow \widetilde{N}$ as in Construction 4.6. Denote the images under the suspension map by $S_{i}^{\prime}, T S_{i}^{\prime} \hookrightarrow \Sigma \widetilde{N} \hookrightarrow \widetilde{N}^{\prime}$. We need to put them in a general position.

First we further describe the topology of $\widetilde{N}^{\prime}$. The manifold $\widetilde{N}^{\prime}$ is a special manifold 3-ad with two distinguished parts of the boundary $\partial_{ \pm} \widetilde{N}^{\prime}=S(V \oplus \mathbb{R}) \times$ $\{ \pm 1\}$. From the decomposition (5.7) we also see that the product $\widetilde{N} \times I$ is a separating submanifold of $\tilde{N}^{\prime}$. By taking its normal disk bundle (using collars) we obtain the codimension 0 embedding $\widetilde{N} \times I^{2} \hookrightarrow \widetilde{N}^{\prime}$, which when restricted to $\widetilde{N} \times\{ \pm 1\} \times I \hookrightarrow \partial_{ \pm} \widetilde{N}^{\prime}$ coincides with the embedding of the closed normal bundle of $\widetilde{N} \times\{ \pm 1\} \hookrightarrow \partial_{ \pm} \widetilde{N}^{\prime}$. In particular there are disks $D_{i}^{ \pm}, T D_{i}^{ \pm}$attached to the normal bundle $\widetilde{N} \times I$ as described in Construction 4.6 See Figure 5 ,

Now denote by $d_{ \pm}: I \rightarrow I^{2}$ the maps $d_{ \pm}(s)=( \pm s, s)$. Using the isotopy induced by the embedding of $\Sigma D_{i}^{ \pm}, \Sigma T D_{i}^{ \pm}$into $\widetilde{N}^{\prime}$ (we omit the details), we can 
change the embedded spheres $S_{i}^{\prime}, T S_{i}^{\prime}$ to embedded spheres in a general position given by the two formulas $S_{i}^{\prime \prime}=\left(D_{i}^{+} \times 1\right) \cup\left(S_{i} \times d_{+} I\right) \cup\left(D_{i}^{-} \times-1\right)$ and $T S_{i}^{\prime \prime}=$ $\left(T D_{i}^{-} \times-1\right) \cup\left(T S_{i} \times d_{-} I\right) \cup\left(T D_{i}^{+} \times 1\right)$. It follows that the associated intersection forms are $\lambda^{\prime}, \mu^{\prime}$, since the only intersections are the intersections in $\widetilde{N}$.

The situation is depicted in Figure 5. The thicker parts correspond to the spheres $S_{i}^{\prime \prime}, T S_{i}^{\prime \prime}$ in a general position. The only intersections are those in $\widetilde{N} \times I$.

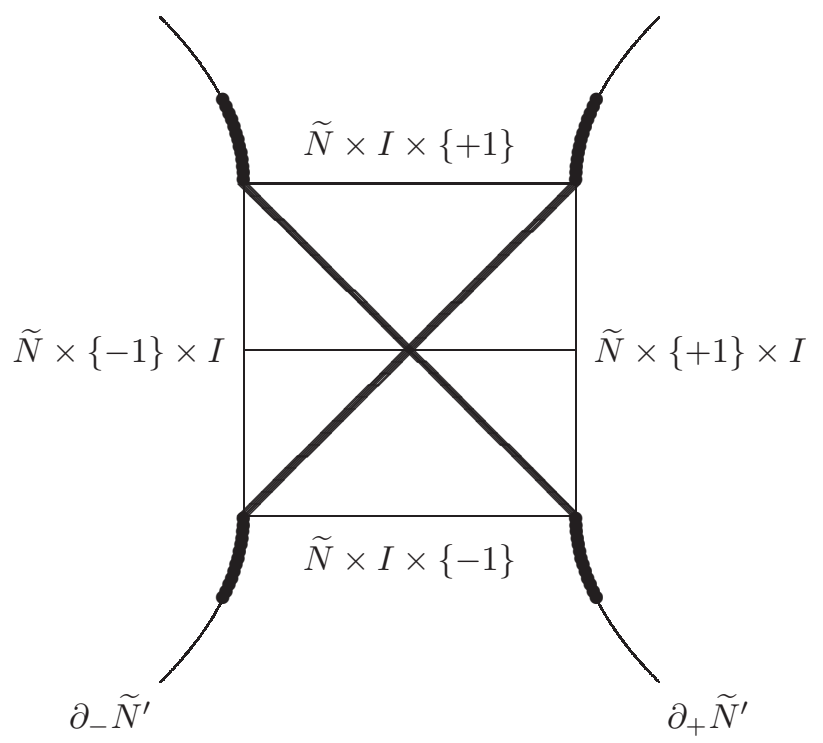

Figure 5. Intersections of the generators of $(H, \lambda, \mu)$ in $\tilde{N}^{\prime}$.

Proposition 5.10. If $F^{(1)}(V, y)$ is not empty, then the map $\tilde{\sigma}_{1}: F^{(1)}(V, y) \rightarrow X_{y}$ is a homotopy equivalence.

Proof. This is proved by showing that the map $\tilde{\sigma}_{1}$ induces isomorphisms on homotopy groups. Note that we have

$$
\pi_{k}\left(F^{(1)}(V, y)\right) \cong \pi_{k}\left(X_{y}\right) \cong L_{k-n}(1) .
$$

Because the surgery obstruction map $\theta$ is an isomorphism, we can realize any element of the group $L_{k-n}(1)$ by a $k$-simplex in $F^{(1)}(V, y)$, that means by an equivariant splitting problem $f: S(V \oplus \mathbb{R}) \times \Delta^{k+1} \rightarrow S(V \oplus \mathbb{R}) \times \Delta^{k+1}$ along $S(V) \times \Delta^{k+1}$ satisfying certain conditions on the boundary.

By Construction 5.3 the image $f^{\prime}=\tilde{\sigma}_{1}(f)$ is a $k$-simplex in $X_{y}$, that means an equivariant splitting problem $f: S\left(V \oplus \mathbb{R}^{2}\right) \times \Delta^{k+1} \times I \rightarrow S\left(V \oplus \mathbb{R}^{2}\right) \times \Delta^{k+1} \times I$ along $S(V \oplus \mathbb{R}) \times \Delta^{k+1} \times I$ again with certain conditions on the boundary.

In order to show that the map $\tilde{\sigma}_{1}$ induces an isomorphism on homotopy groups, it is enough to show that if the splitting obstruction $\theta(f)$ is represented by a simple $(-1)^{m}$-hermitian form $\left(H^{+}, \lambda_{0}, \mu_{0}\right)$, where $2 m=k-n$, then the splitting obstruction $\theta\left(f^{\prime}\right)$ is represented by the same simple $(-1)^{m}$-hermitian form. But this is just a version of the statement that was proved in a sequence of Lemmas 5.7 5.8, 5.9 parametrized over $\Delta^{k+1}$. The proofs carry over to the parametrized case as well. 
Theorem 5.11. We have

$$
\pi_{k}\left(\Theta F^{(1)}\right) \cong L_{k}(1) \text { for } k \in \mathbb{Z},
$$

where the groups $L_{k}(1)$ are the 4-periodic L-groups from surgery theory associated to the trivial group.

Proof. Note that the proof of Proposition 5.5 tells us in the special case $y=*$ that modulo some low-dimensional deviations the spectrum $\Theta F^{(1)}$ is an $\Omega$-spectrum and we know its homotopy groups. Namely

$$
\pi_{k}\left(\Theta F^{(1)}\right) \cong \pi_{k+|k|+8}\left(F^{(1)}\left(\mathbb{R}^{|k|+8}\right)\right) \cong L_{k}(1)
$$

for all $k \in \mathbb{Z}$.

\section{ACKNOWLEDGEMENTS}

The present paper grew out of my thesis defended at the University of Aberdeen. I would like to thank my supervisor Michael Weiss for conversations and encouragement during the work on this project and my thesis referees Michael Crabb and Andrew Ranicki for useful comments. My thanks are also to Mamuka Jibladze for conversations and for the pictures in the paper. Finally I would like to thank the Max Planck Institut für Mathematik in Bonn for their hospitality during the final stages of the preparation of this paper.

\section{REFERENCES}

[AH] D R Anderson, W-C Hsiang, 'The functors $K_{i}$ and pseudoisotopies of polyhedra', Annals of Mathematics 105 (1977), pp. 201-223. MR0440573 (55:13447)

[Ar] G Arone, 'The Weiss' derivatives of $\mathrm{BO}(-)$ and BU(-)', Topology 41 (2002), pp. 451-481. MR.1910037 (2003c:55012)

[BK] A K Bousfield, D M Kan, Homotopy limits, completions and localizations (LNM 304, Springer-Verlag, Berlin, New York, 1972). MR0365573 (51:1825)

[Br] W Browder, 'Free $\mathbb{Z}_{p}$ actions on homotopy spheres', in "Topology of manifolds. Proc. 1969 Georgia Conference." Markham Press, 1970, 217-226. MR0276982 (43:2720)

[BL] W Browder, G R Livesay, 'Fixed point free involutions on homotopy spheres', Bull. Amer. Math. Soc. 73 (1967), 242-245. Also Tohoku J. Math. 25 (1973), 69-88. MR0206965 $(34: 6781)$

[BLR] D Burghelea, R Lashof, M Rothenberg, Groups of Automorphisms of Manifolds (SLN 473, Springer-Verlag, 1975). MR0380841(52:1738)

[BS] J C Becker, R E Schultz, 'Equivariant Function Spaces and Stable Homotopy Theory I', Comment. Math. Helv. 94 (1974) pp. 1-34. MR0339232 (49:3994)

[Cr] M C Crabb, $\mathbb{Z}_{2}$-Homotopy Theory (LMS Lecture Note Series 44, CUP 1980). MR0591680 (83m:55010)

[Dw] W G Dwyer, 'Classifying Spaces and Homology Decompositions', preprint, Notre Dame, 1998.

[Go] T Goodwillie, 'Calculus III, Taylor tower', Geometry and Topology (2003), 645-711. MR2026544 (2005e:55015)

[Ha] I Hambleton, 'Projective surgery obstructions on closed manifolds', in Algebraic Ktheory. Proc. Oberwolfach 1980. Part II. LNM 967, Springer, 1982, 101-131. MR0689390 (84g:57026)

[KS] R C Kirby, L C Siebenmann, Foundational Essays on Topological Manifolds, Smoothings, and Triangulations (Princeton University Press, Princeton, NJ, 1977). MR0645390 (58:31082)

[LdM] S López de Medrano, Involutions on manifolds (Ergebnisse der Mathematik und ihrer Grenzgebiete 59, Springer-Verlag, New York-Heidelberg, 1971). MR0298698 (45:7747) 
[Lu] W Lück, A Basic Introduction to Surgery Theory (SFB 478 Preprint-Reihe, University of Münster, http://wwwmath.uni-muenster.de/math/inst/sfb/ about/publ/heft197.ps, 2001).

[Mac] T Macko, Ph.D. Thesis, University of Aberdeen, 2003.

[MW] T Macko, M Weiss, 'The block structure spaces of real projective spaces and orthogonal calculus of functors II', in preparation.

[MR] I Madsen, M Rothenberg, 'On the classification of G-spheres. II. PL-automorphism groups', Math. Scand. (2) 64 (1989), 161-218. MR1037458 (91d:57024)

[Mad] I Madsen, 'On the space of manifold structures for lens spaces', Indian J. of Math. 25 (1983), 277-304. MR0809260 (87j:57020)

[Mi] J Milnor, Lectures on h-cobordism Theorem, notes by L. Siebenmann and J. Sondow (Princeton University Press, Princeton, NJ, 1965). MR0190942 (32:8352)

$[\mathrm{Qu}]$ F Quinn, 'A Geometric Formulation of Surgery Theory', in Topology of Manifolds (Proc. Inst., Univ. of Georgia, Athens, GA, 1969), Markham, Chicago, IL, 1970, pp. 500-511. MR0282375 (43:8087)

[Ra1] A A Ranicki, Exact Sequences in the Algebraic Theory of Surgery (Princeton University Press, Princeton, NJ, 1981). MR0620795 (82h:57027)

[Ra2] A A Ranicki, Algebraic L-theory and Topological Manifolds (Cambridge Tracts in Math. 102, Cambridge University Press, 1992). MR1211640 (94i:57051)

[RS] C P Rourke, B J Sanderson, ' $\Delta$-sets I: Homotopy theory', Q. J. Math. Oxford (2) 22 (1971), 321-338. MR0300281 (45:9327)

[Wa] C T C Wall, Surgery on Compact Manifolds (2nd edition, edited by A A Ranicki, Mathematical Surveys and Monographs 69, AMS, 1999). MR1687388(2000a:57089)

[We] M Weiss, 'Orthogonal Calculus', Transactions of the AMS 347 (1995), 3743-3796. MR.1321590 (96m:55018)

[WW] M Weiss, B Williams, 'Automorphisms of Manifolds', in Surveys on Surgery Theory, Annals of Mathemathics Studies 149, Princeton University Press, 2001, pp. 165-220. MR.1818774(2002a:57041)

Mathematisches Institut, Universität MÜnster, Einsteinstrasse 62, Münster, D-48149, Germany - AND - MatematickÝ Ústav SAV, Šteffánikova 40, Bratislava, SK81473, SLOVAKIA

E-mail address: macko@math.uni-muenster.de 